\title{
Mars ionospheric response to solar wind variability
}

\author{
H. J. Opgenoorth, ${ }^{1,2,3}$ D. J. Andrews, ${ }^{1}$ M. Fränz, ${ }^{3}$ M. Lester, ${ }^{4}$ N. J. T. Edberg, ${ }^{1}$ \\ D. Morgan, ${ }^{5}$ F. Duru, ${ }^{5}$ O. Witasse, ${ }^{6}$ and A. O. Williams ${ }^{4}$ \\ Received 20 March 2013; revised 19 August 2013; accepted 23 August 2013; published 3 October 2013.
}

[1] At planets with induced magnetospheres, the coupling between the ionosphere, the weak draped magnetosphere, and the solar wind is very direct in comparison to Earth. The weak induced magnetosphere itself is created by the prevailing Solar wind conditions and therefore in its shape and strength dynamically depending on it. In early 2010, Mars was located behind Earth in the Solar wind; thus, we can use coordinated data from multiple near-Earth spacecraft (Stereo, Wind) to evaluate what kind of Solar wind disturbances have passed by Earth and might consecutively hit Mars, and when. We employ plasma data from the ESA Mars-Express mission, the ASPERA-3 particle instrument, and the MARSIS Active Ionospheric Sounder (AIS) to investigate, for a number of isolated events in March and April 2010, how the ionosphere and the induced magnetosphere at Mars develop and decay in response to Solar wind variability in the magnetic field, density, and velocity. In a dedicated campaign mode, we use frequent long-duration MARSIS AIS operations for several consecutive orbits, to monitor for the first time the long-term development of the Martian plasma environment during solar wind disturbances. We find that the magnetosphere and ionosphere of Mars can become considerably compressed by solar wind dynamic pressure variations, which usually are also associated with changes in the magnetic draping of the interplanetary magnetic field around the planet. These are typically associated with corotating interaction regions and coronal mass ejections, and can last for several days. During such episodes of compression, we see signatures of increased plasma transport over the terminator and enhanced ion outflow from the upper atmosphere.

Citation: Opgenoorth, H. J., D. J. Andrews, M. Fränz, M. Lester, N. J. T. Edberg, D. Morgan, F. Duru, O. Witasse, and A. O. Williams (2013), Mars ionospheric response to solar wind variability, J. Geophys. Res. Space Physics, 118, 6558-6587, doi:10.1002/jgra.50537.

\section{Introduction}

[2] Planets or planet-like bodies without intrinsic magnetic fields are considered to lose considerable fractions of their atmosphere in a more or less permanent dynamic process of erosion occurring in the interaction between the ionized upper atmosphere and the flowing solar wind plasma (or in the case of moons, the plasma flow in the host planet's magnetosphere). In the case of Mars, this atmospheric ion loss has been estimated to be of the order of $0.05-0.5 \mathrm{~kg} \mathrm{~s}^{-1}$ or $10^{23}-10^{24}$ ions s $^{-1}$ [see, e.g., Barabash et al., 2007; Lundin et al., 2008a; Edberg et al., 2010; Nilsson et al., 2010, and

\footnotetext{
${ }^{1}$ Swedish Institute of Space Physics, Uppsala, Sweden.

${ }^{2}$ International Space Science Institute, ISSI, Bern, Switzerland.

${ }^{3}$ Max-Planck-Inst. f. Sonnensystemforschung, Katlenburg-Lindau, Germany.

${ }^{4}$ Department of Physics and Astronomy, University of Leicester, Leicester, UK.

${ }^{5}$ Department of Physics, University of Iowa, Iowa, USA.

${ }^{6}$ ESA/STEC, Noordwijk, Netherlands.

Corresponding author: H. J. Opgenoorth, Swedish Institute of Space Physics, Box 537, SE 75121 Uppsala, Sweden. (opg@irfu.se)

(C)2013. American Geophysical Union. All Rights Reserved. 2169-9380/13/10.1002/jgra.50537
}

references therein]. Several potential loss processes such as bulk ion loss, ionospheric ion outflow, and solar wind pickup have been advocated, which all are associated with direct solar wind forcing. Thermal processes have played a dominant role in the early life of the planet Mars. They are probably now much less dominant, except in the case of hydrogen escape, but still comparable to present day nonthermal loss processes [Lammer et al., 2013].

[3] However, a few studies concerning the influence of solar wind variability, in particular solar wind dynamic pressure changes caused by changes either in plasma density, velocity, or both, have shown that the intensity of the outflow is strongly dependent on such solar wind parameters. Lundin et al. [2008b] have shown that the outflow rate is dependent on both solar wind dynamic pressure and the EUV/XEUV flux. Futaana et al. [2008] showed that a burst of Solar Energetic Particles (SEP) associated with a coronal mass ejection (CME) caused a short-lived rise of the atmospheric escape rate at Mars by a factor of 10 . Results of these studies have been reviewed by Dubinin et al. [2011].

[4] However, such studies of direct solar wind trigger of outflow events at Mars are rare, as there is no readily available upstream solar wind monitor at Mars, which could provide information on the state of the solar wind, while lowaltitude Mars orbiting satellites measure these substantial 


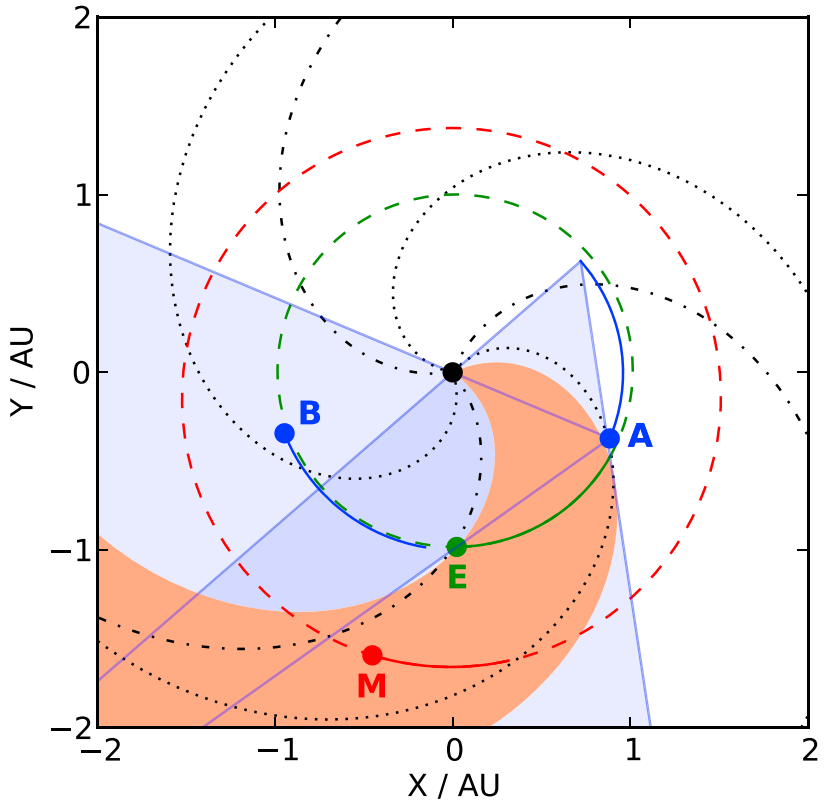

Figure 1. The trajectories of Earth (E, green), Mars (M, red), and Stereo (blue, A-ahead and B-behind Earth) as colored dashed lines in "Heliocentric inertial" coordinates from 1 March 2010 to 1 May 2010. Colored solid circles indicate the position of the respective planet or spacecraft at the start of this interval, while the solid circular segments indicate their motion to the end of the interval. Black dotted and dashed lines show sets of nominal Parker spirals corresponding to fast $\left(600 \mathrm{~km} \mathrm{~s}^{-1}\right)$ and slow $\left(300 \mathrm{~km} \mathrm{~s}^{-1}\right)$ solar wind streams, respectively. The orange-shaded region indicates the area swept out by Parker spirals which intersect the orbit of Earth, corresponding to the average solar wind speed measured by Wind during the whole interval, $\sim 420 \mathrm{~km} \mathrm{~s}^{-1}$. Furthermore, the blue-shaded areas in this figure demark the STEREO-A Secchi HI fields of view from Sun to Earth (and Mars) at the beginning and the end of the campaign period, as used in Figure 2.

changes in the energies of the relevant plasma populations inside the bow shock (BS) (i.e., within the heated magnetosheath plasma of the induced magnetosphere) and within the magnetic pileup boundary (MPB) (i.e., within the colder and typically less dense planetary ionosphere). To overcome the absence of a dedicated upstream solar wind monitor at Mars, Nilsson et al. [2010] used the combined data set of ESA's Mars Express (MEX) and NASA's Mars Global Surveyor (MGS) in a statistical study, confirming the dependence of observed ionospheric outflow rates, observed by the former, to solar wind variations of dynamic pressure, observed by the latter. In a first detailed event study, Dubinin et al. [2009] investigated the effects on the Martian ionosphere for one clear corotating interaction region (CIR) event, which resulted in a major scavenging of the Martian ionosphere and an increase in the ion outflow by a factor of 10. In this special case occurring in February 2008, Mars was located just behind Earth along the solar wind Parker spiral, and thus solar wind data at Earth (from the ACE spacecraft) could be used to predict the impact of a CIR at Mars (see our own campaign description below). They interpreted detailed observations of ionospheric plasma heating by the ASPERA-3 and MARSIS instruments on MEX (see more detailed instrument description and references below) as a consequence of plasma penetration from the hot magnetosheath plasma to inside the MPB, leading to significant particle acceleration and bulk ion outflow for only a few hours of the duration of the initial impact of the CIR front. In a similar study, Wei et al. [2012] compared the effect of a CIR on the ion outflow from Earth and Mars when both planets were radially aligned in January 2008.

[5] Another example of such rare event studies has been published by Edberg et al. [2009b]. They made use of the favourable coincidence of a Rosetta Mars flyby, which occurred in close temporal relation to an impact of a CIR on the Martian ionosphere. Thus, they could use the Rosetta magnetometer and plasma instruments (which had been switched on during this flyby for calibration and testing purposes) in the solar wind in the vicinity of Mars to determine solar wind parameters, which could have caused the associated changes in magnetosheath population and ionospheric plasma escape from Mars as seen by the ASPERA-3 and MARSIS instruments on MEX. Also, they found a sudden appearance of energetic heavy ionospheric ions, with energies increasing with altitude, along a dawn-dusk orbit of MEX following the terminator. Before and after the event, these accelerated ions were not observed at all, and already on the second orbit following the passage of the CIR they were observed at a much lower rate. One should note that during both case studies above the coverage of the MARSIS topside Active Ionospheric Sounder data (AIS) was relatively poor, as the instrument was operating within the generally prioritized subsurface sounder mode (see below).

[6] Based on such results from rare event studies, Edberg et al. [2010] carried out a follow-up statistical study based on ACE observations of 41 high-pressure events during clear-cut CIRs (purposely avoiding the more complicated CME signatures) from the solar minimum period of 2007-2008, including the Earth-Mars solar wind conjunction period in early 2008. Comparing all available ASPERA-3 energy bins, they observe a statistical increase of accelerated heavy ion outflow rates by a factor of at least 2.5 , between times of CIR impact and non-CIR solar wind conditions. Their results furthermore confirm the compression of the magnetosheath ion population and the planetary ionosphere below during the passage of solar wind pressure pulses. However, they note that such comparisons between disturbed and quiet times are difficult as the overall data coverage is not the same in both conditions, and in particular the overall magnetospheric compression during disturbed times might lead to more concentrated outflow patterns, affecting the correct calculation of average outflow rates.

[7] On the basis of such previous results, a group of scientists within the so-called Mars Upper Atmosphere Network (MUAN, a loosely organized group of researchers involved in Martian plasma physics using MEX, MGS, and, in the near future, MAVEN data) decided in late 2009 that one should try to overcome the absence of upstream solar wind monitoring at Mars by coordinated effort rather than hoping for more fortuitous events supporting scattered event studies. This paper is intended to demonstrate the superior potential of coordinated multispacecraft instrument operation, as the first of hopefully many resulting studies from a unique data set during solar minimum conditions, which emerged from a special coordinated campaign. 


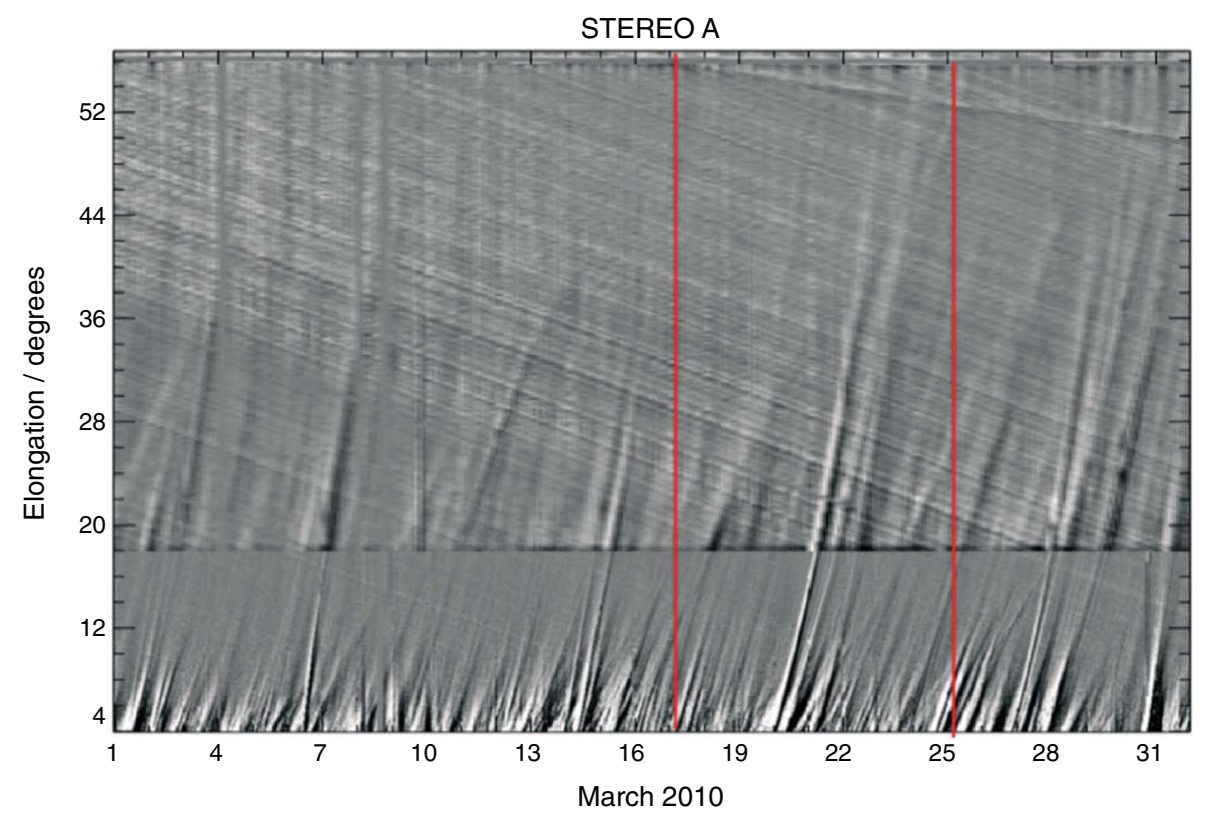

Figure 2. So-called J-plots of solar wind density structures, projected on to the Sun Earth line, based on Stereo A Secchi HI observations (see text for more details). Red vertical lines indicate the approximate arrival estimates for two distinct groups of solar ejecta at Mars, discussed as events 1 and 2 below. The inclined track from $54^{\circ}$ elongation on 17 March to $50^{\circ}$ on 31 March 2010 is the track of planet Mars, while the horizontal track at exactly $56^{\circ}$ is planet Earth. Other (more inclined) tracks stem from insufficiently removed stellar sources.

\section{Campaign Overview and Instrumentation}

\subsection{Heliospheric Configuration During March and April 2010}

[8] Accurate knowledge of the upstream solar wind conditions is a central requirement for any investigation into the instantaneous state of the Martian induced magnetosphere. Here, the intermittent monitoring of the solar wind by the ASPERA-3 instrument during the outermost portion of MEX's orbit is supplemented through the use of continuous measurements made by the Wind spacecraft orbiting at the L1 point at Earth. During March-April 2010, Earth and Mars were approximately aligned along the Parker spiral, such that, at least for relatively constant sources of solar wind variability, such as corotating interaction regions, measurements of solar wind bulk parameters made at one planet can be justifiably extrapolated to the other [Vennerstrøm et al., 2003], as will be discussed in section 2.2. In addition, we employ data from the wide-field heliospheric imager on the STEREO spacecraft, discussed in section 2.3.

[9] Figure 1 shows the orbits of Earth (E, and therefore the Wind spacecraft, in green), Mars (M, and therefore MEX, in red), and STEREO A (ahead of Earth in its orbit) and B (behind, both in blue), during the period of this "campaign" from 1 March to 1 May 2010. Their motion is depicted in the inertial Heliospheric Earth Ecliptic frame, rotated for purposes of presentation such that Earth is located at $X=0$ at the beginning of the interval. Two sets of nominal Parker spirals are also shown, corresponding to slow $\left(300 \mathrm{~km} \mathrm{~s}^{-1}\right.$, dotted line) and fast ( $600 \mathrm{~km} \mathrm{~s}^{-1}$, dot-dashed line) solar wind speeds. The orange-shaded region indicates the area swept out by Parker spirals corresponding to the average solar wind speed measured by Wind during the whole interval, $\sim 420 \mathrm{~km} \mathrm{~s}^{-1}$, which intersect the orbit of Earth. Furthermore, the blueshaded areas in this figure demark the STEREO HI fields of view from Sun to Earth (and Mars) at the beginning and the end of the campaign period.

\subsection{Solar Wind Data at $1 \mathrm{AU}$, Wind, and STEREO}

[10] We use data from the STEREO spacecraft in order to more accurately distinguish the nature of the structures seen to propagate in the solar wind to Mars during the campaign. The two STEREO spacecraft were launched into heliocentric orbits, such that they would separate from Earth at a rate of $22^{\circ}$ per year. The STEREO A (ahead) leads the Earth in its orbit, while the STEREO B (behind) follows it; thus, during the first years of their lifetime, they provided a stereoscopic view of solar ejecta toward Earth. Each spacecraft carries a variety of in situ and remote sensing instruments, in particular the Sun-Earth Connection Coronal and Heliospheric Investigation (SECCHI) of which we use the Heliospheric Imagers (HI) [Eyles et al., 2009]. Two off-set coronagraphs HI-1 and HI2 , looking along the Sun-Earth line, allow for the observations of solar ejecta close to the sun from $4^{\circ}$ to $18^{\circ}$, and further out in the heliosphere toward $\sim 1 \mathrm{AU}$ from $4^{\circ}$ to $57^{\circ}$ viewing angle. During the time of our campaign, the STEREO spacecraft were already too widely spaced to provide real stereoscopic viewing capability, although Figure 1 shows that the STEREO A spacecraft was ideally located on the eastern (leading) side of the sun, to monitor solar ejecta directed toward Earth (and even Mars) at this time. On the other hand, one can also see from this figure that in situ measurements at both STEREO A and B are obviously not directly related to the solar wind disturbances arriving at either Earth or Mars.

[11] In Figure 2, we show the observations during the month of March 2010, from the two HI cameras on STEREO A, 


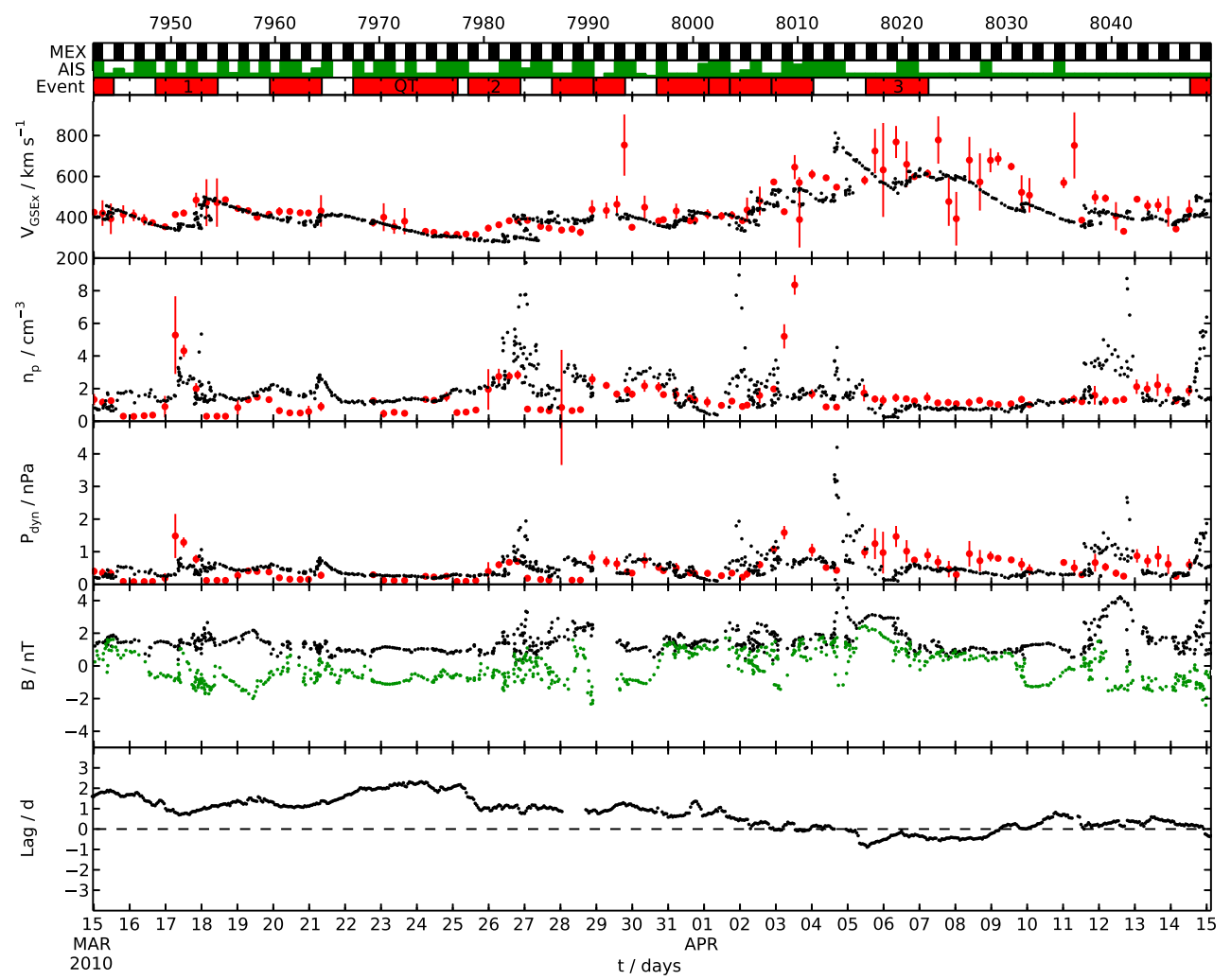

Figure 3. Campaign overview plot illustrating from top to bottom, (a) orbit numbers, (b) MARSIS AIS orbital coverage, (c) event identification and naming, (d) solar wind velocity from Wind measurement at Earth, propagated and scaled toward Mars (black dots) and plasma velocities as seen by ASPERA-3 outside the Martian bows hock (red dots, one value per apoapsis portion of each orbit, with the variability indicated by a vertical red "error bar," see text for more detail), (e) solar wind density from Wind and Mars Express (as Figure 3d)), (f) solar wind dynamic pressure resulting from the data in Figures 3d and 3e. (g) Total (black) and radial (green) magnetic field as seen by Wind at $1 \mathrm{AU}$, propagated and scaled to Mars (see text for details). (h) Delay between observations at $1 \mathrm{AU}$ and estimated arrival time at Mars, following assumed propagation along Parker spiral arm, with observed solar wind velocity at $1 \mathrm{AU}$.

using a technique of creating time-versus-elongation maps of solar wind density variations (so-called "J-maps"), first used for SOHO data by Sheeley et al. [1999]. As y axis, we use the direction away from the earthward pointing limb of the sun, in the ecliptic plane, where we plot the observed solar ejecta projected onto the Sun Earth line (see Williams et al. [2011] for more details on this technique, and compare also to Figure 1).

[12] To create this image, differenced observations are used to artificially highlight faint propagating electron density features, resulting in white leading and black trailing edges indicating, respectively, increases and decreases in solar wind electron density. It becomes evident from Figure 2 that there are many tracks apparent at various angles, indicating solar wind density structures propagating with various velocities away from the sun. Other features visible are the boundary of overlap between the HI- 1 and HI- 2 imagers at $18^{\circ}$, the trace of Earth, stable at $56^{\circ}$ in the top of the figure, and the trace of Mars, in this projection passing behind Earth into visibility of the HI-2 imager on 17 March 2010. There are also stripes in the background, which result from incompletely suppressed star light tracks. For the purpose of this study, we draw attention to two sets of multiple, converging structures emerging from the Sun on 14 or 15 March and on 20 March, which are seen to reach the elongation of the projection of Earth and Mars at around 17 and 25 March, respectively.

[13] Such converging "families" of tracks of density structures are characteristic signatures of CIRs in the HI data, and they correspond to plasma elements that have been emitted by the same corotating source region on the Sun at different times and have subsequently been entrained at the stream interface [Roulliard et al., 2010; Williams et al., 2011]. However, one has to take great care in identifying signatures of quasi-steady sources like CIRs, in which the almost purely radial motion of the plasma in the solar wind, combined with the rotation of its source on the solar surface, leads to the formation of so-called Parker spiral structures. These resemble a sweeping spiral segment with a leading high-density interplanetary shock, as indicated in Figure 1. Knowing the arrival time of such a spiral segment at one planet allows the prediction of the arrival time of another very similar spiral segment, stemming from the same solar source region, at another planet. In contrast, coronal mass ejections (CMEs) are short-lived, violent, and sporadic phenomena, with often much higher plasma ejection velocities. Thus, their signature in the solar wind is more like a spatially confined "blob" of radially expanding high-velocity plasma. embedded in closed magnetic structures. Thus, the exact arrival time of 
CME structures at planets is very difficult to predict from far upstream data sources and depends on the actual CME event itself, its spatial limitations, its direction, and its consequent interaction with the solar wind plasma, which is overtaken in the progress of the interplanetary CME expansion..

[14] Inspecting Figure 1 and comparing to the bottom panel in Figure 3 below, it becomes obvious that CIR structures reach Earth before Mars during the beginning of the campaign, while they reach Mars before Earth during the later campaign, when Mars was located further behind Earth in their rotation around the Sun. For fast expanding CME-like structures, on the other hand, the relative arrival time will strongly depend on where the central portion of the CME is located. If, for example, there is no perfect radial alignment between the planets (and during this campaign, we concentrated on a time with planetary alignment along the Parker spiral), a CME, which hits Earth with its central part, can engulf Mars in the plasma at the CME flank, which arrives later than predicted from the motion of the leading CME shockfront (see our discussion below, and also the illustrative Figure 1 of Rouillard et al. [2010] for further details).

\subsection{Wind Measurements of Solar Wind Parameters}

[15] In addition to remote sensing imagery using STEREO, we also employ in situ data from the NASA Wind spacecraft orbiting at the Earth-Sun L1 point, 0.01 AU upstream of Earth. Specifically, we use hourly averages of the solar wind bulk velocity and density from the Solar Wind Experiment [Ogilvie et al., 1995], noting that this instrument is expected to be able to provide reliable measurements even during the passage of a particularly tenuous parcel of solar wind observed during the campaign, as will be later discussed in section 3.1. In addition, for survey purposes, we employ similar hourly averaged data from the Wind Magnetic Field Investigation [Lepping et al., 1995] to check for significant, maintained rotations in the heliospheric field that would indicate extended crossings of the heliospheric current sheet.

[16] These data are time shifted and appropriately scaled to form a continuous prediction of the upstream solar wind conditions at Mars throughout the campaign, and are shown in Figure 3 (along with in situ measurements in the vicinity of Mars, just outside the Martian bow shock, made using the ASPERA-3 instrument onboard MEX where possible, as introduced in section 2.4). Specifically, following Vennerstrøm et al. [2003] and Williams et al. [2011], we apply a time shift $\Delta t$ to the measured Wind solar wind data, which is given by

$$
\Delta \mathrm{t}=\frac{\Delta \mathrm{r}}{v_{r}}+\frac{\Delta \theta}{\Omega_{s}}
$$

where $\Delta r$ is the radial separation between Earth and Mars, $\Delta \theta$ the corresponding azimuthal separation, $\Omega_{\mathrm{S}}$ is the equatorial solar rotation period, and $v_{r}$ is the radial speed of the solar wind. The value of $\Delta t$ is computed for each hourly average independently, and we choose not to perform any averaging or other filtering processes to the time-shifted data, such that the periods for which this simple geometric propagation becomes uncertain are readily visible as those periods in Figure 3 for which the time-shifted quantities are no longer single valued. This indicates intervals for which we must expect some evolution of the solar wind between Earth and
Mars, as faster parcels of solar wind plasma interact with preceding slower ones. The solar wind density measured at Earth shown in Figure 3 has additionally been scaled (downward) to correspond to the distance of Mars, under the assumption of conservation of mass-flux through the heliosphere. This downscaling has a corresponding influence on the solar wind dynamic pressure values shown. Finally, we note that the quantity $\Delta t$, explicitly shown in the lower panel of Figure 3, can on occasion be negative, when Mars lags behind Earth in its orbit by a significant amount, and thus gets engulfed in a sweeping spiral arm earlier than Earth does (see discussion above and illustration in Rouillard et al. [2010]).

\subsection{In Situ Solar Wind Measurements at Mars Using MEX}

[17] In addition to the time-shifted solar wind data, we also show corresponding measurements, made using the ASPERA-3 instrument on MEX [Barabash et al., 2006], whenever the spacecraft was in the solar wind, i.e., close to the apocenter of every orbit. The ASPERA-3 experiment onboard MEX consists of a suite of sensors making both in situ and remote measurements, specifically the Ion Mass Analyser (IMA), the Electron Spectrometer (ELS), and two Energetic Neutral Atom (ENA) detectors [Barabash et al., 2006]. IMA measures composition $(\mathrm{m} / \mathrm{q})$, energy $(E / q)$, and angular distribution of ions in the energy range of $10 \mathrm{eV}$ to $30 \mathrm{keV}$, with a field of view of $90^{\circ} \times 360^{\circ}$, achieved by electrostatic scanning in the elevation direction. Measurements of the cold/low-energy ion component $(E \leq 50 \mathrm{eV})$ are carried out without the elevation scan, with a corresponding field of view of $4^{\circ} \times 360^{\circ}$. The achieved time resolution for the measurements of the cold and hot ion components is thus $16 \mathrm{~s}$ and $192 \mathrm{~s}$, respectively. The ELS sensor measures a two-dimensional distribution of electron fluxes in the energy range of 5 to $20 \mathrm{keV}$ with a time resolution of $4 \mathrm{~s}$ and a field of view of $4^{\circ} \times 360^{\circ}$. When the instrument is in the solar wind, the density and bulk flow speed of solar wind ions is estimated via numerical fits to these distributions [Fränz et al., 2006]. In panels $\mathrm{d}$, e, and $\mathrm{f}$ of Figure 3, we show in addition to the propagated Wind data ASPERA-3 measurements of the solar wind speed, density, and dynamic pressure in red. These data, owing to the significant scatter often present in the ASPERA-3 distribution function fits, have been plotted only as one singular mean value of each parameter as determined during each excursion of MEX into the solar wind, spanning the apoapsis portion of each orbit. Vertical bars show \pm 1 standard deviation uncertainties similarly determined.

\subsection{Summary of Solar Wind Variations Seen During the Campaign}

[18] In summary, Figure 3 shows, from top to bottom, three ancillary panels giving (a) the MEX orbit designation as rows of alternate black and white blocks, (b) green staples indicate the fraction of data from the MARSIS Active Ionospheric Sounder (AIS) obtained on the corresponding orbit (the height of each block being proportional to the number of successful soundings obtained, the reader is referred to a full description of the instrument and our data presentation in section 2.6 below), and (c) red blocks indicating the naming and duration of the individual "events" discussed in section 3 . Five data panels then follow, each comprised of timeshifted data appropriate to the study of the upstream 

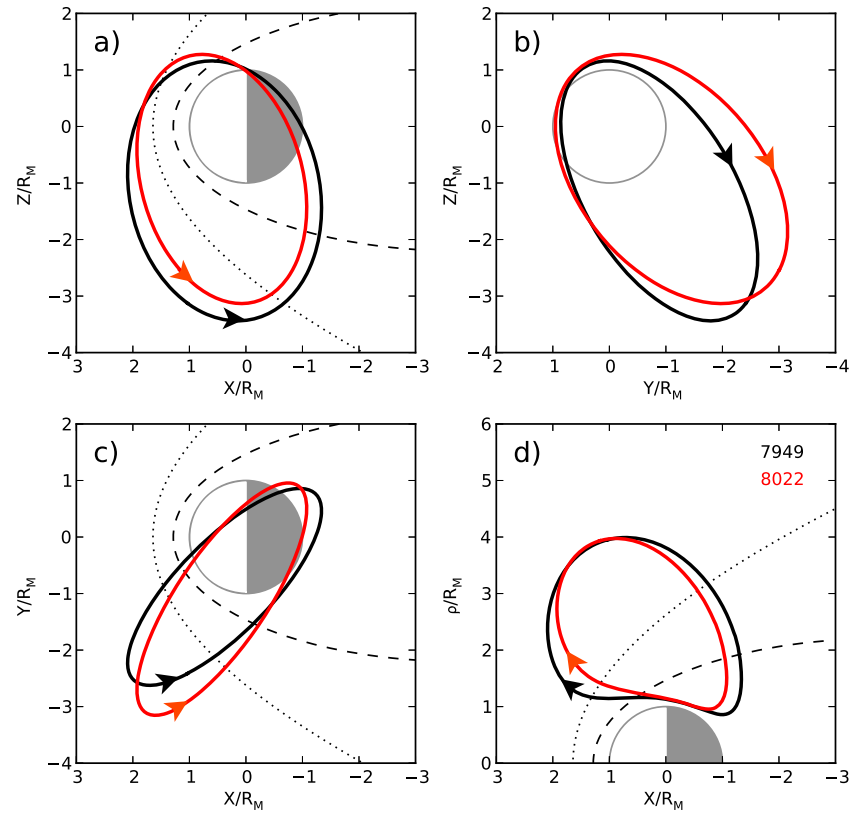

Figure 4. Mars Express orbit at the beginning (black orbit 7949 on 15 March 2010) and end (red orbit 8022 on 7 April 2010) of the MUAN Earth Mars conjunction campaign in (a) the MSO Z/X plane, (b) Z/Y plane, (c) YX plane, and (d) cylindrical coordinates (see text for details).

conditions at Mars. Panel d) shows Wind and ASPERA-3 solar wind velocities in the radial direction as black and red circles, respectively, including \pm 1 standard deviation uncertainties for the ASPERA-3 values, discussed in the next section. Panel e) shows corresponding solar wind proton densities in the same format as panel $d$ ). It should be noted that ASPERA-3 typically underestimates the solar wind density for periods when solar wind temperature is low. Panel f) shows solar wind dynamic pressure determined from the measurements shown in panels d) and e). Panel g) shows the total magnetic field (black) and the radial field component (green) measured by Wind at L1, time shifted and scaled to correspond to an interplanetary magnetic field (IMF) which, somewhat tentatively, can be expected to arrive at Mars. Finally, panel h) shows the time shift $\Delta t$, which has been applied to the above data from the Wind spacecraft at $1 \mathrm{AU}$.

[19] From the complex data gathered during this period, we would like to highlight four intervals of interest, which we will discuss in more detail in section 3 below. On 17 March, there is a strong rather short-lived increase in the solar wind density detected in both the time-shifted Wind data and in the in situ measurements made by ASPERA-3. It is followed by a longer lasting increase in the solar wind velocity at Earth and Mars. At the same time, the magnetic field has some sharp excursions, followed by a period of higher variability. Such signatures are a characteristic mark of the encounter of a CIR event as shown, e.g., by Tsurutani et al. [1995], or Edberg et al. [2010], in a superimposed epoch analysis of 41 CIRs (see their Figure 2). We will hereafter refer to this as "Event 1" (see red block 1 in panel c) of Figure 3 ).

[20] On 25-26 March, there is another very similar event seen, with a long-lived velocity increase associated with a short-lived increase in the solar wind density and magnetic field. Again, the magnetic field clearly has a higher variability following this event, which we will refer to as "Event 2." We note that these two most prominent events during our campaign are associated with the approximate arrival times of the STEREO CIR traces in Figure 2 (marked by red vertical lines), originating from the Sun on 14-15 and 20 March 2010, respectively.

[21] A third event of interest for this particular study occurs around 5-6 April, when Wind observes increased electron density and solar wind velocities exceeding $800 \mathrm{~km} \mathrm{~s}^{-1}$ at $1 \mathrm{AU}$ in the morning of 5 April, predicting an arrival of similar activity at Mars late on 4 April, on the assumption of CIR type of propagation. However, the picture at Mars is somewhat more complex, as we note that ASPERA-3 does not measure elevated densities until 6 April. As a matter of fact, this disturbance at $1 \mathrm{AU}$ is caused by a major $\mathrm{CME}$, which fortuitously, being the first major CME of the then newly rising solar cycle, has already been studied in detail by Rouillard et al. [2010] during its propagation to Earth. There it was most probably responsible for the loss of the Canadian Galaxy 15 satellite [Allen, 2010]. This CME occurred late in the campaign, at a time when the propagation of Parker spiral-shaped CIR features would reach Mars just before or at the same time when they affected Earth (see negative or zero delay in the bottom panel h) of Figure 3). As the main body of this CME expanded very fast radially toward Earth from the Sun, this event reached Mars clearly not before 6 April 2010, when ASPERA-3 detects a clear increase in the solar wind velocity (see more detailed text below in section 3.4). In the following, we will refer to this CME as "Event 3."

[22] In addition to these events of particular solar wind disturbances, we note that during 22-24 March, there is an extended period of extreme quiescence in the solar wind, coincident with plasma densities dropping below $\sim 2 \mathrm{~cm}^{-3}$. At this time, Solar wind velocities were only at or below $300 \mathrm{~km}$ $\mathrm{s}^{-1}$, resulting in a dynamic pressure of less than $0.3 \mathrm{nPa}$. In section 3.1 below, we will discuss this particularly quiet interval as a baseline, in order to characterize the undisturbed plasma environment at and around Mars, in contrast to our observations during the other three chosen examples of active solar wind disturbances. In panel c) of Figure 3, we mark this period as QT. All remaining unlabeled red bars in this panel refer to other events under study during this interval, but are not subject of this paper.

\subsection{MEX Data Within the Induced Martian Magnetosphere}

[23] The MEX spacecraft is in a highly eccentric orbit around Mars with a periapsis altitude of $\sim 275 \mathrm{~km}$, an apoapsis of $\sim 10,000 \mathrm{~km}$, and an inclination of $\sim 86^{\circ}$ [for a detailed description of the MEX mission, see Chicarro et al., 2004]. Figure 4 shows the MEX orbit in three orthogonal planes in Mars solar orbital (MSO) coordinates (panel a-c), and in a cylindrical coordinate system (panel d), for two orbits corresponding to the beginning and end of the campaign interval presented here. Modeled positions of the magnetic pileup boundary and the bow shock, according to Vignes et al. [2000], are also shown. We see that during the time of this dedicated conjunction campaign the MEX orbit was such that the spacecraft entered the Martian 
magnetosheath in the southern tail, continued into the nightside upper ionosphere before reaching periapsis near the northern dawn flank terminator region and finally exited through the magnetosheath on the dayside dusk flank, returning back into the upstream solar wind. Furthermore, it becomes clear that the orbit configuration changes only slowly throughout the ca. 70 orbits in this campaign. Thus, the amount of change between any shorter sequence of orbits, as investigated during the event studies below, is so small that we are able to directly compare data from such orbits without introducing any spatial ambiguities. Similar and comparable regions will be passed from orbit to orbit. The orbit geometry at the time of the campaign is particularly well suited for the study of dayside bow shock and magnetospheric compressions, trans-terminator plasma transport, nightside ion outflow, and flank compression and tail-flank flaring processes. This particular geometry actually happens to be similar to the orbit geometry encountered during the event studied by Dubinin et al. [2009], but it is different from the along-terminator periapsis orbit geometry at the time of the Rosetta Mars flyby studied by Edberg et al. [2009b].

[24] Throughout this campaign, ASPERA-3 was operated in an optimized mode, which, however, is very close to the nominal mode of permanent operation. ASPERA-3 can make measurements along the entire orbit and thus monitors both electron and proton distributions in the solar wind (see section 2.4 above), and hot/cold electron and typically a mixture of planetary and solar wind ion distributions in the Martian magnetosheath and ionosphere, respectively.

[25] The MARSIS Active Ionospheric Sounder (AIS) shares the $40 \mathrm{~m}$ antenna of the MEX spacecraft with the Subsurface Sounder (SS) designed to look for subsurface signatures of ice and water remnants [Jordan et al., 2009]. During AIS operation, short radio wave pulses are emitted at 160 frequencies, quasi-logarithmically spaced and increasing from $\sim 0.1$ to $\sim 5.5 \mathrm{MHz}$. The delay time from transmission to reception of any corresponding echo is then measured. Such echoes can be caused either by the Martian ionosphere at altitudes where the ionospheric plasma density is such that the radio pulse is specularly reflected, or by reflections from the Martian surface itself (in the case of emitted radio frequencies which lie above the plasma frequency corresponding to the maximum ionospheric electron density). The resulting frequency-delay curve is usually called an "ionogram." It can then be numerically inverted to form an altitude profile of the ionospheric plasma density [see, e.g., Morgan et al., 2008]. Typically, the MARSIS topside sounder only yields feasible results over regions of the Martian ionosphere with peak densities above $\sim 10^{4} \mathrm{~cm}^{-3}$. Each such frequency sweep takes $\sim 1.5 \mathrm{~s}$ to complete, with successive sweeps being performed every $\sim 7.5 \mathrm{~s}$.

[26] The amount and duration of MARSIS AIS operation are usually determined by the requirements of the subsurface sounder instrument, which uses the same antenna. A socalled "full orbit" operation of the MARSIS AIS spans the time of the closest approach of Mars Express to Mars, from about $1200 \mathrm{~km}$ altitude, past periapsis and back to $1200 \mathrm{~km}$ altitude on the outbound leg, which lasts for typically 44 min on each orbit. During this campaign, MARSIS AIS data were taken substantially more frequently than is generally the case, so as to afford the most complete understanding of the response of the Martian ionosphere to passing solar wind disturbances, which can take place over several successive MEX orbits (several tens of hours). However, owing to various constraints, this rate was still often reduced as is depicted by the green blocks in the upper portion of Figure 3, panel b). Full blocks indicate a 44 min operation period around pericenter, approximately half-full blocks refer to AIS operation on the dayside outbound segment of the orbit below $\sim 1200 \mathrm{~km}$, while approximately quarter-full blocks indicate one or two short segments of AIS operation between 1200 and about $500 \mathrm{~km}$ altitude. The campaign-related enhanced rate of AIS soundings can be seen to dramatically decrease after about 7 April, as the instrument is returned to its nominal subsurface priority of operation, after the last event discussed below. However, in retrospect, we note that an optimum of 5-15 consecutive full AIS orbits would have been necessary to cover entire (and even multiple) events of enhanced Mars solar wind interaction, and one should thus aim at even more continuous AIS operations for future campaigns.

[27] Beyond the capability of topside ionospheric sounding, MARSIS is also able to provide useful in situ measurements. Fortuitously, through direct interactions between the MARSIS antennas and the local plasma, excitations occurring at the local plasma frequency (and higher harmonics thereof) produce significant distortion signals in the instrument, from which the local plasma density can be retrieved. This phenomenon happens under certain conditions, namely within the Martian magnetic pileup boundary where the plasma temperature is expected to be sufficiently low, such that the "cold plasma dispersion relation" will hold. Furthermore, the bulk flow speed of the plasma needs to be sufficiently low, such that these distortions remain close to the antennas long enough to be measured [Gurnett et al., 2005; Duru et al., 2008]. The latter condition imposes a practical upper limit of $\sim 100 \mathrm{~km} \mathrm{~s}^{-1}$ to the bulk speed for which the plasma density can be measured, therefore unfortunately precluding measurements of density during passages through the magnetosheath and in the solar wind. Similarly, sometimes harmonic "pseudo-echoes" are received by the instrument, which are spaced at multiples of the local electron gyroperiod. Whenever occurring, these can provide good estimates of the magnetic field magnitude, for fields ranging over a few nT to $\sim 150 \mathrm{nT}$ [Gurnett et al., 2005]. This measurement is of particular scientific value, as MEX possesses no dedicated magnetometer.

[28] Furthermore, we note that along with these actual MARSIS measurements of key plasma parameters, sometimes even the absence of data, such as the absence of ground echoes, local plasma lines or electron-gyroperiod echoes also can provide some information regarding the state of the Martian induced magnetosphere. For example, absence of echoes from the Martian surface can be a signature of ionospheric radio absorption due to the presence of a collisional low-altitude ionospheric layer as may be produced during SEP events [Morgan et al., 2010]. Similarly, sudden local plasma density depletions by compressions of the ionosphere to altitudes below the spacecraft, or alternatively fast plasma motion at spacecraft altitude as encountered during magnetosheath passages, may at times inhibit the detection of the excited plasma resonances. Thus, any such signature of "no data" can still be used as indirect proxy for the characterization of the prevailing plasma conditions in the ionosphere around or below the spacecraft. 


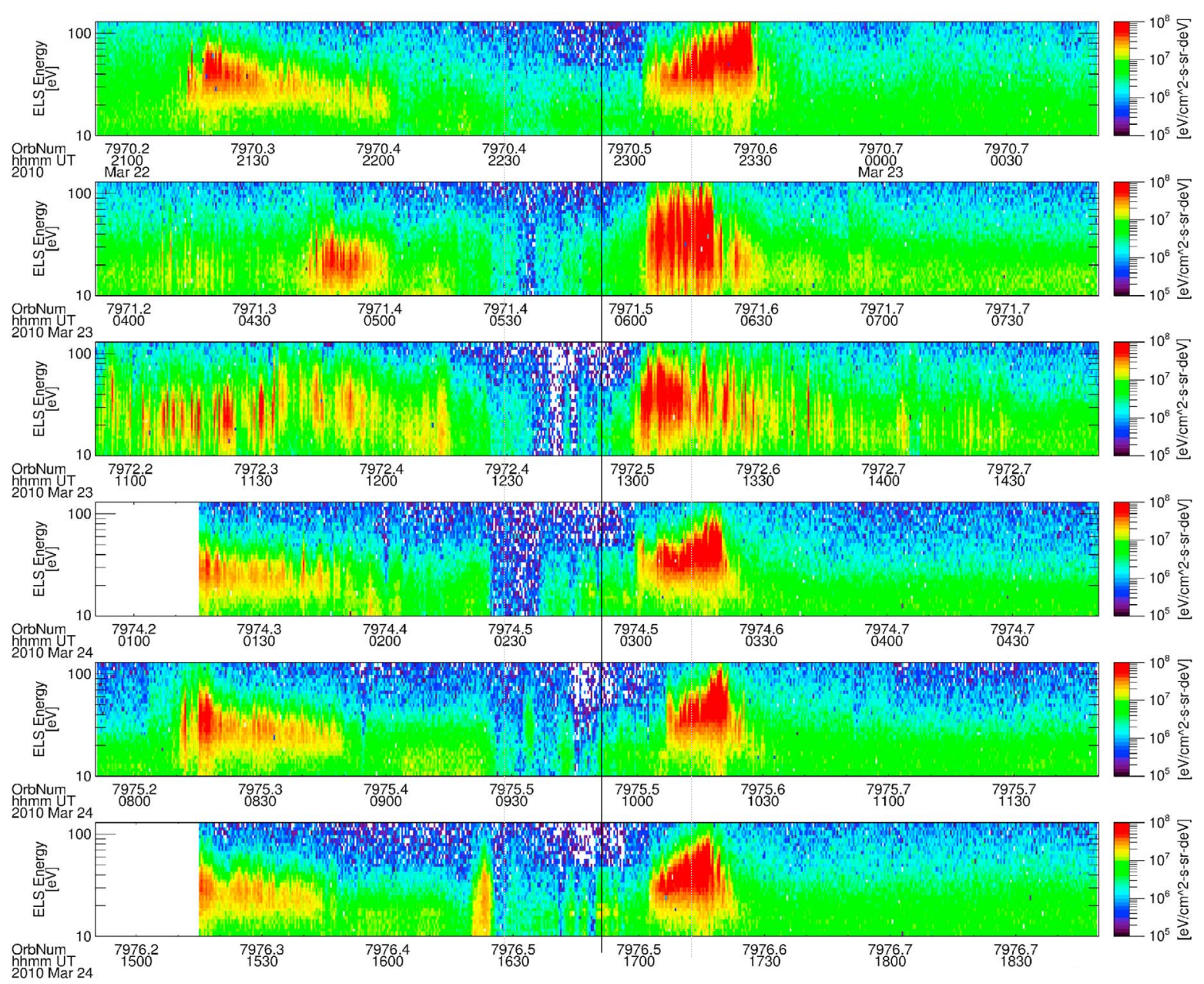

Figure 5a. Sequence of ASPERA-3 electron energy spectra along six consecutive orbits for the quiet time period 22-24 March 2010 (orbits 7970, 71, 72, 73 malfunction, 74, 75, 76). The data panels for each orbit span time intervals of $4 \mathrm{~h}$, centred around pericenter (black vertical line), which is sufficient to cover solar wind, magnetosheath, ionosphere, and the exit through the dayside magnetosheath, back into the solar wind (see text for more details). Vertical grey dotted lines demark the potential coverage of simultaneous MARSIS AIS data (44 min centered around pericenter).

\section{Observations From ASPERA-3 and MARSIS During Selected Time Periods}

\subsection{The Quiet State of the Induced Magnetosphere of Mars (22-25 March 2010)}

[29] As already discussed, the period of March 2010 is relatively quiet in terms of active disturbances on the Sun, and the three selected events, which are discussed below in sections 3.2 to 3.4, are caused by relatively isolated solar events in a generally "solar minimum" type of activity level. However, even in this overall very quiet campaign period, the interval from 22 to 25 March stands out as particularly calm and void of any clear solar wind activity. During this interval, marked by the red block "QT" at the top of Figure 3, the Wind data (time shifted to Mars) suggest a steady solar wind, with a velocity of $\sim 300 \mathrm{~km} \mathrm{~s}^{-1}$, densities below $2 \mathrm{~cm}^{-3}$, and a resulting solar wind dynamic pressure of less than $0.3 \mathrm{nPa}$. The variability of the IMF is very low throughout this time interval.

[30] In order to illustrate the state of the induced magnetosphere and the ionosphere of Mars at such generally quiet conditions, we show in Figures $5 \mathrm{a}$ and $5 \mathrm{~b}$ sequences of panels of the ASPERA-3 electron and ion spectrograms, respectively. Specifically, Figure 5 shows data around the pericenter of orbits 7970 to 7976 (22 March, 21:00 to 24 March, 18:50 UT). As noted in the previous section, the slow evolution of MEX's orbit over the campaign allows ASPERA-3 data taken on the periapsis passage of consecutive orbits to be directly compared with each other in terms of stemming from basically the same regions revisited from orbit to orbit. Therefore, such stacked data sets can illustrate temporal changes in the local state of the prevailing plasma, and differences in the spatial configuration of the boundaries between solar wind and magnetosheath, the magnetic pileup boundaries, and the ionosphere around Mars. The spectrogram panels are stacked by ascending orbit number, with the periapsis in each case at the center of the panel (marked by a vertical black line). In any such panel (and for all similar figures below), the presented ASPERA-3 data extend for $4 \mathrm{~h}(2 \mathrm{~h}$ in each direction from pericenter), thus allowing to safely observe the entire Martian magnetosphere and ionosphere from Solar wind exit on the flank to reentry on the dayside. 


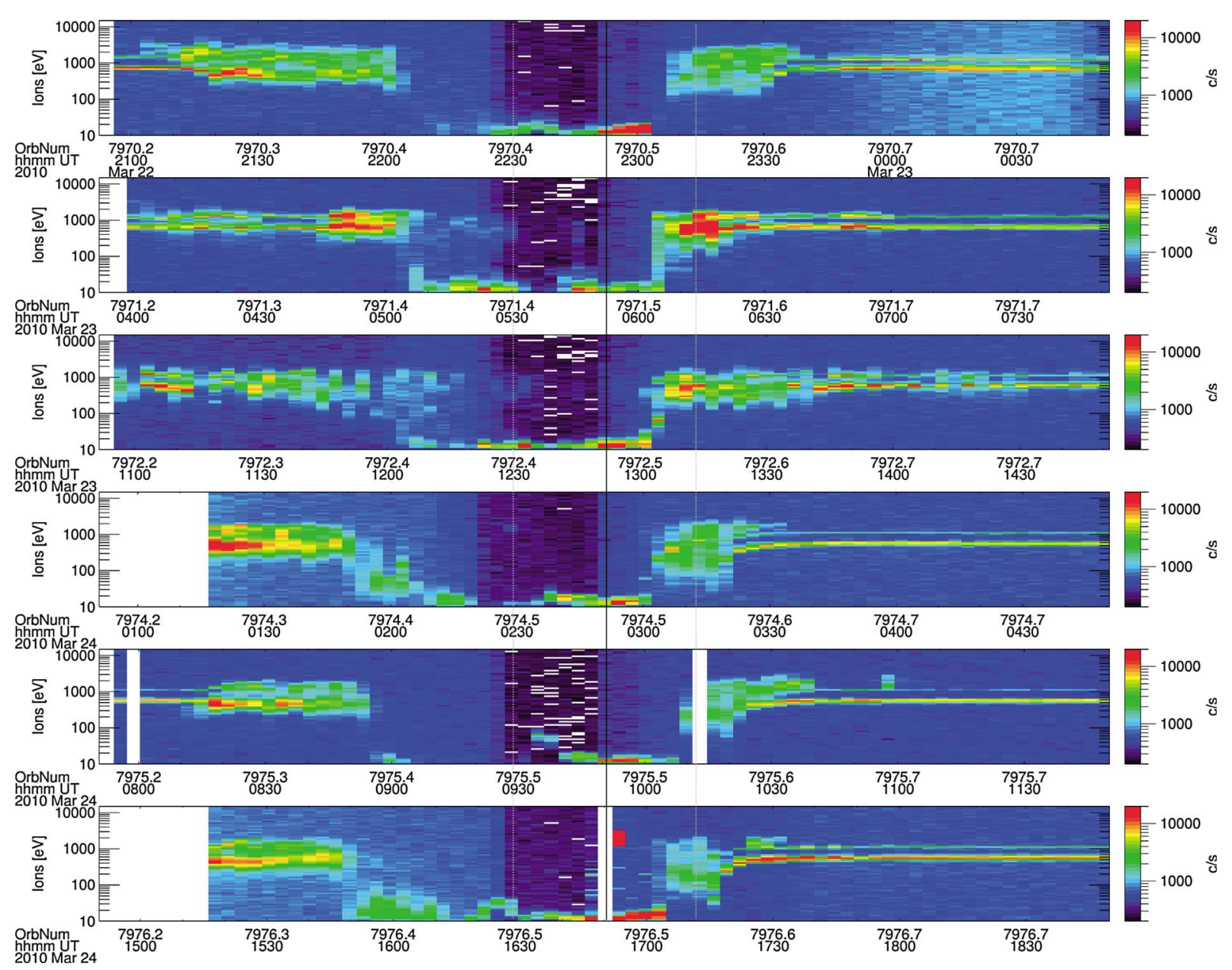

Figure 5b. Sequence of ASPERA-3 all ion energy spectra for the quiet time period 22 to 24 March 2010 (for orbits 7970, 71, 72, 73 malfunction, 74, 75, 76). Else as Figure 5a.

[31] In this way, the time coordinate can be taken as a proxy for spatial distances along the slowly changing orbital trajectory (compare to Figure 4). As each orbit is roughly $7 \mathrm{~h}$ apart, a virtual long-term time axis (of the order of hours and days for these figures) runs down the ordinate of the complete set of stacked plots. The ordinates of each individual panel are logarithmic particle energies, and the color scale represents the particle energy flux at $8 \mathrm{~s}$ resolution for electrons and the count rate at $192 \mathrm{~s}$ resolution for ions.

[32] In detail, the electron spectra in the uppermost panel of Figure 5a (orbit 7970 on 22 March 2010) thus illustrate the passage of the spacecraft from the low-density solar wind on the dawnside tail of the Martian magnetosphere to a moderately denser and somewhat warmer magnetosheath, behind the flank bow shock, which is encountered at about 21:15 UT. Shortly after 22:05, MEX enters the ionospheric cavity (or nightside plasma wake) behind the magnetic pileup boundary (MPB) where the observed electrons are mainly photo-electrons. The spacecraft leaves the ionosphere again at 23:05 UT, now on the dayside, after passing periapsis at about 22:50 UT. Again, a hotter and denser magnetosheath population is present, which is seen until the spacecraft enters the solar wind again at 23:30 UT. In the stacked sequence of electron spectra from orbit to orbit, this general situation does not appear to change for the next few days until orbit 7976 on 24 March 2010. (Note that there is one orbit, 7973 on 23 March without data, lacking in this stack). The relative position of the MPB and magnetosheath boundaries does not change much during this entire period. The only visible change is that the magnetosheath particles get less well defined by their energy range as Mars ionosphere slowly adjusts to the quiet time period of the Solar wind from orbit 7970 to 7972 (first three panels).

[33] The respective ion spectra at $192 \mathrm{~s}$ resolution for the same orbits in Figure 5b show essentially the same spatial plasma behavior and location of the prime boundaries between solar wind, magnetosheath, and ionosphere, as the electrons in Figure 5a. In addition, one can clearly see the population of suprathermal $(<20 \mathrm{eV})$ ionospheric ions, located between the inner magnetosheath plasma boundaries, which are characterized by a clear step in ion energies from $\sim \mathrm{keV}$ in the magnetosheath to several $\mathrm{eV}$ in the ionosphere. The magnetosheath ion population is denser (higher count rate) and has a wider spread of energies (temperature) than the solar wind at the far left and right of each panel. One can also note considerably higher counts of suprathermal ions in the dayside ionosphere.

[34] Using the mass-spectrometer capabilities of ASPERA3 , the solar wind ions can be separated from the higher mass ions of ionospheric origin. In Figure 5c, we therefore display only data from the ASPERA-3 heavy ion mass channels above 


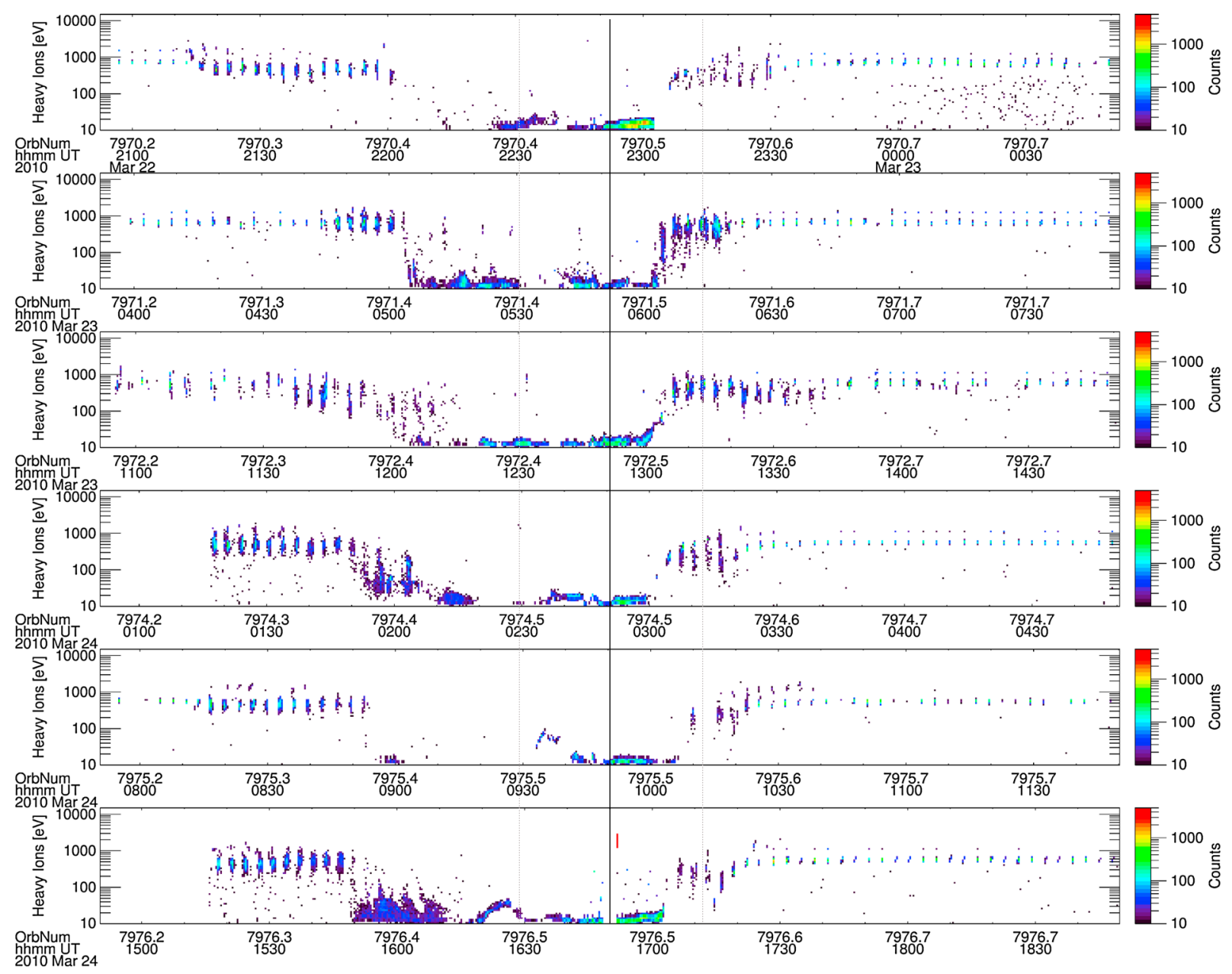

Figure 5c. Sequence of ASPERA-3 heavy ion energy spectra for the quiet time period 22 to 24 March 2010 (for orbits 7970, 71, 72, 73 malfunction, 74, 75, 76). Else as Figure 5a.

the Helium ion mass in highest time resolution (12s). One can see that the low-altitude low-energy ion population consists entirely of heavy ionospheric ions. However, a more detailed examination of the mass-spectrometer data from the original energy-mass matrixes (see, e.g., Barabash et al. [2006], data not shown here) shows that the apparent high-energy heavy ion populations in the solar wind and magnetosheath regime stem from proton contamination into the high-energy highmass spectrometer channels, while the low-energy ions are clearly $\mathrm{O}_{2}^{+}$and $\mathrm{O}^{+}$of ionospheric origin.

[35] In both sequences of ion spectra around orbital pericenters (for all ions in Figure $5 \mathrm{~b}$ and for heavy ions in $5 c)$, it can again be seen that the definition of the Martian magnetosheath and the clarity of boundaries between the initially well-defined plasma regimes get more and more diffuse the longer the solar wind remains quiet. The entire magnetosphere appears to widen both on the nightside flank (to the left of periapsis) and on the subsolar dayside (to the right of periapsis). Toward the end of the quiet period, and in particular during orbit 7974 and 7976, the nightside observation of moderately energized heavy ions (few tens of eV) increases somewhat, but there is no clear acceleration of ions with altitude (distance from periapsis), as is typically seen during more active times (see all other ion data in the active events below). This effect can best be seen in the heavy ion data on the left side of Figure 5c (panels 4 and 6, for orbits 7974 and 7976). We note that a similar acceleration of topside ionospheric plasma was reported by Pérez-de-Tejada et al. [2009] in the region between the magnetosheath and the ionosphere, which they identified as a velocity boundary layer, stemming from eroded ionospheric plasma. From the data presented here, it seems that this process may be active even during very quiet solar wind conditions.

[36] Apart from this minor increase in the nightside suprathermal ionospheric ion population, the transitions between magnetosheath and ionospheric ions at the inner MPB are sharply marked by an increase of the mean energy from $10-20 \mathrm{eV}$ in the ionosphere to $0.8-1.2 \mathrm{keV}$ in the magnetosheath within one $192 \mathrm{~s}$ sampling window. The ion energy distribution along orbit resembles a rectangular "boxcar" function, with well-defined step-like boundaries between two distinct ion populations. These boundaries are at the nominal locations of the MPB. This distinct separation has been further confirmed by detailed inspection of the mass-resolved data from IMA. In order to illustrate one more time the actual locations in space of these data, and the absence of any energetic heavy ions along the entire orbit, we use in Figure $5 \mathrm{~d}$ a presentation of the integrated heavy ion density in the energy range from 50 to $200 \mathrm{eV}$ along the MEX orbit 7970 in cylindrical coordinates (integration of data from the first panel in 


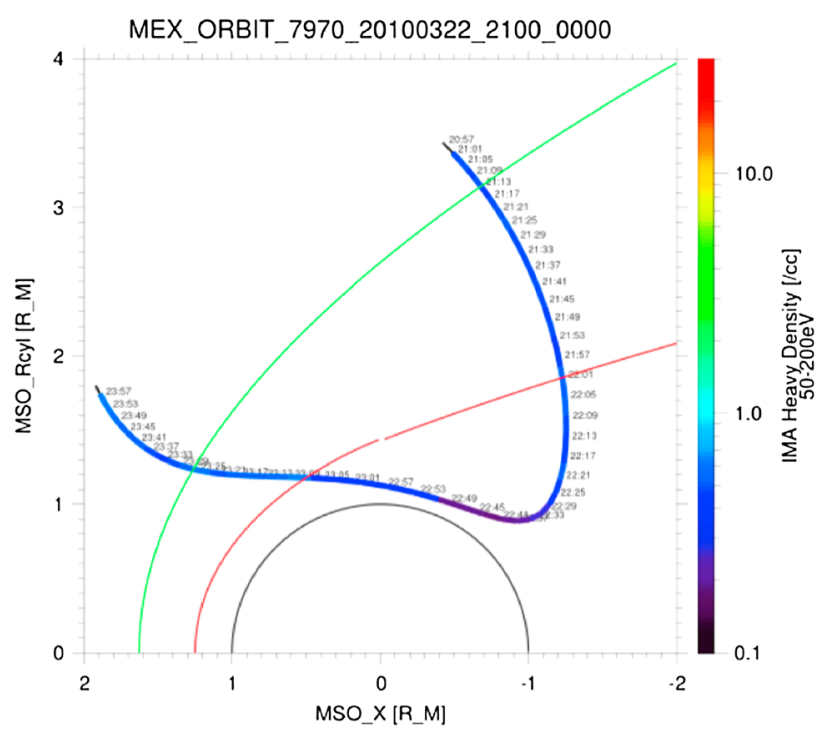

Figure 5d. Integrated local density of heavy ions in the 50-200 eV energy range in cylindrical coordinates for orbit 7970 on 22 March 2010.
Figures $5 \mathrm{~b}$ and $5 \mathrm{c}$ ). As can be seen, and in contrast to average results from more disturbed conditions by, e.g., Edberg et al. [2009a] and Barabash et al. [2007], the density of such ions is less than $0.5 \mathrm{~cm}^{-3}$ anywhere along the orbit.

[37] After characterizing the shape and location of the magnetosheath and ionospheric boundaries in the ASPERA3 data, we would now like to inspect the MARSIS data for particular features characterizing a quiet Martian ionosphere. From the green blocks in the second panel of Figure 3, we see that during the beginning of this period of quiet and steady solar wind, we were lucky to have, in fact, two consecutive MARSIS-AIS passages throughout the entire closest approach to Mars. In Figure 6, we show AIS data for orbits 7970 and 7971 in a particularly designed overview format, which we will also use to characterize the ionospheric dynamics during more active events below. We have plotted in the top panel of Figure 6 the electron densities at spacecraft altitude, as derived from the local plasma resonances seen in the AIS ionograms (black dots representing data from orbit 7970 and red dots for orbit 7971). We also mark periapsis by the vertical dotted line, which during the campaign period fortuitously happens to almost coincide with the optical terminator for solar illumination of the topside ionosphere. In the second panel,

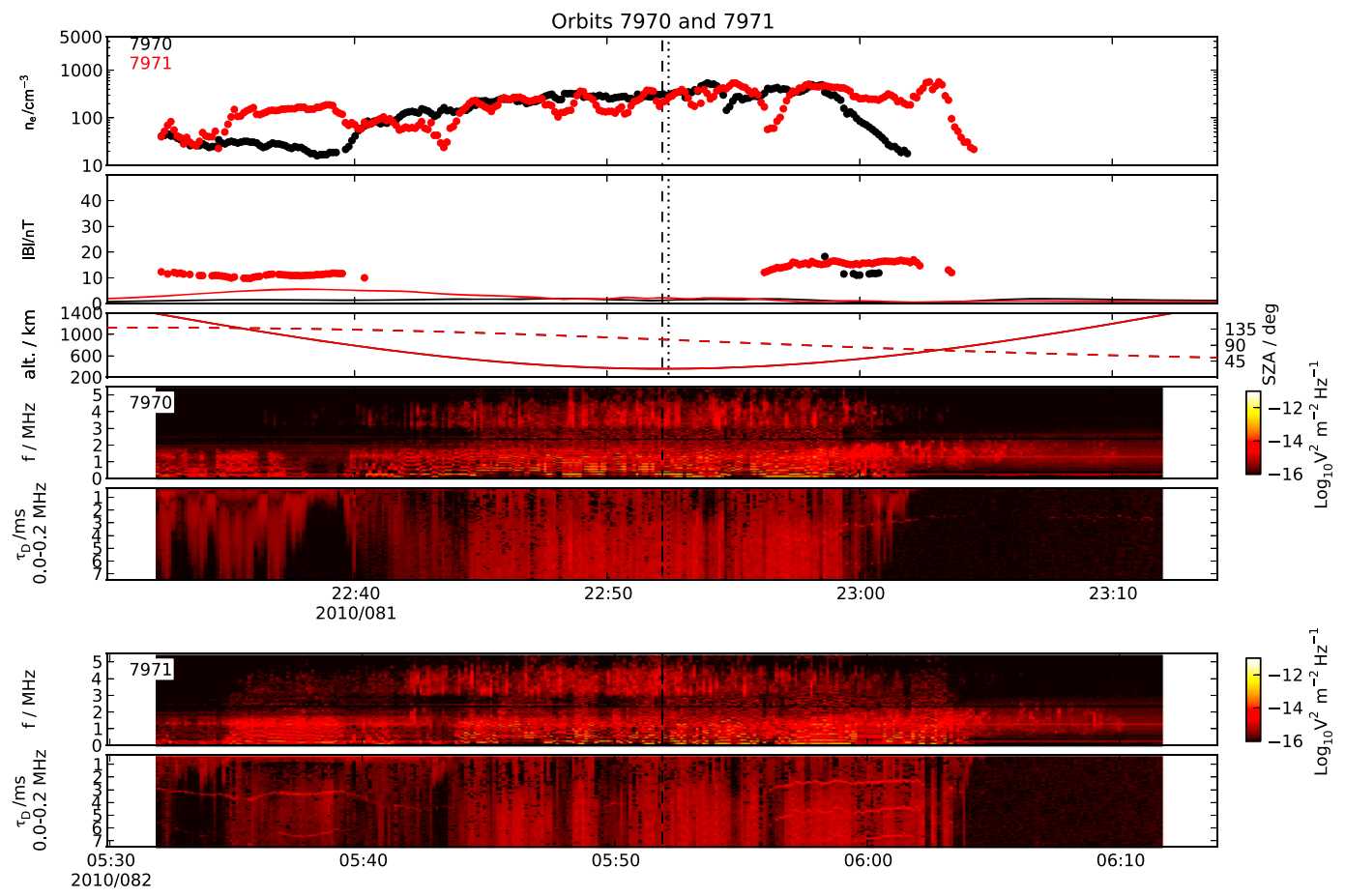

Figure 6. Plots of MARSIS AIS data from the quiet time period, on orbits 7970 and 7971. First panel: Local electron density for orbit 7970 (black) and 7971 (red), measured |B| (solid colored circles using the same color scheme), Second panel: $|\mathrm{B}|$ derived from the Cain et al. [2003] model (thin curves using the same color scheme). Here, data from the lattermost orbit have been time shifted by the MEX orbit period to overly those taken earlier. Third panel: MEX altitude (solid line) and solar zenith angle (SZA, broken line), then followed by two pairs of panels for the two orbits, showing in each case AIS data averaged in delay in the upper panel of the respective pair (highlighting the local plasma frequency oscillations), and averaged over low frequencies ( $<<0.2 \mathrm{MHz}$ ) highlighting the electron gyroperiod echo. Vertical dashed and dotted lines indicate the positions of the terminator for the first and second orbits, respectively. The data span 44 min centered around the pericenter. 
we present the estimates of the total magnetic field strength at spacecraft altitude, as derived from the observations of electron gyrolines in the ionograms (again black dots refer to data from orbit 7970, and red dots to orbit 7971).

[38] In the following four panels, we visually present the general behavior of the series of MARSIS AIS data taken throughout these two orbits. As AIS ionograms are derived every $7.5 \mathrm{~s}$, one cannot easily display their temporal and spatial sequence in straightforward graphics. The upper of each of these two panels per orbit in the middle and bottom of Figure 6 shows integrated MARSIS data in which average echo intensity is color coded and plotted versus sounding frequency and time (c.f., Duru et al. [2008], note that we show the same type of data for orbit 7970 and 7971). In such a frequency versus time presentation, the presence of local plasma oscillations is seen as horizontal "stripes" at lower frequencies (generally below $\sim 2 \mathrm{MHz}$, with larger vertical spacing indicating higher densities). One can also see the more steady ground echo at the frequencies above $\sim 3 \mathrm{MHz}$, while the presence of an ionospheric reflection in this presentation of the data becomes evident as a brighter, but still diffuse signal at intermediate frequencies in the dayside portion of the orbit, in each case. The rise and fall in frequency of such echoes allow us to at least qualitatively understand variations in the maximum ionospheric density below the spacecraft with respect to solar zenith angle, which increases toward the terminator. The maximum frequency of these echoes (indicating the peak ionospheric electron density) is typically seen to decrease from the right side of the figure toward the center.

[39] The second panel in each such pair represents a similar section through the ionograms, but orthogonal to the one chosen above, in which the received signals across all frequencies below $0.2 \mathrm{MHz}$ are simply averaged [c.f., Gurnett et al., 2005]. We choose to display integrated data from the lowest frequency bin as it typically contains the strongest signal of electron gyrolines, visible in this format as horizontal lines, the spacing between which is inversely proportional to the local magnetic field strength. Here, the diffuse signal intermittently present at all delays is due to the local plasma oscillations. Such a (low-frequency) delay-versus-time diagram allows us in the first place to evaluate the validity of the total magnetic field $\mathrm{B}$ measurements displayed in the second panel of this figure, and it helps considerably to visualize the actual presence of local plasma lines, in contrast to the other panel, which integrates all delays for each frequency bin, such that ground echoes and ionospheric echoes often add to the plasma-line signal.

[40] After this necessarily complicated introduction of the data presentation in Figure 6, let us now discuss what we actually see in this data. In the same way as in Figure 5, we would like to interpret the data in the context of changes in plasma parameters in space rather than in time. However, as already mentioned above, the MARSIS AIS data are only received for times when the Mars Express orbit is below $1200 \mathrm{~km}$ altitude, which spans approximately $44 \mathrm{~min}$ around periapsis. In order to allow a more direct comparison of such data with the $4 \mathrm{~h}$ span of simultaneous ASPERA data from the same region, we mark in all relevant figures (i.e., Figure 5 above and Figures 7, 9, and 11 below) the span of the MARSIS AIS data with two grey dotted lines, spaced $22 \mathrm{~min}$ on each side of pericenter, which is marked by a full black line. As in all other figures, the nightside part of the orbit is to the left of Figure 6, and the dayside on the right.

[41] Keeping this in mind and looking at the MARSIS data in Figure 6 from right to left (i.e., moving against the time axis from the day to the nightside in the Martian magnetosphere), we note that the lattermost point at which we can determine local electron density using plasma-line echoes for orbit 7970 lies at about 2305 UT, which is the same location as the inner edge of the magnetosheath plasma in the ASPERA-3 data in Figure 5. After an initial steep rise of electron density toward lower spacecraft altitudes (again moving from right to left) in the figure, the density then levels out for the entire lower portion of the orbit, past the terminator, and only decreases somewhat toward the nightside portion of the ionosphere, indicating a considerable plasma convection over the terminator from the dayside sunlit to the nightside dark ionosphere. The local electron density remains relatively stable, but decreasing, until the AIS operations terminate in the deep nightside of the planet at $\sim 1200 \mathrm{~km}$ altitude, corresponding to $\sim 2230$ UT on the time axis. At this time, the ASPERA data in Figure 5 still show that the spacecraft is engulfed by ionospheric electrons and ions until at least 2200 UT (moving in negative time direction along the orbit).

[42] The magnetic field estimate during this orbit (black dots in second panel of Figure 6) is consistent with a draped solar wind IMF of $\sim 12 \mathrm{nT}$ at an altitude of $600 \mathrm{~km}$ above the dayside ionosphere, which is relatively weak in comparison to observations during more active solar wind conditions (see below). We note here, for further discussion below, that the observation of the magnetic field strength at such locations only indicates the absolute amount of the IMF draping around the ionospheric obstacle, thus neither the shape nor the direction of the actual draping. A detailed description of the IMF draping geometry around Mars has, for example, been given by Bertucci et al. [2003], based on full vector magnetic field observations by the Mars Global Surveyor magnetometer. Furthermore, no detection of such gyrolines by MARSIS does not necessarily mean a low magnetic field, but could also be the consequence of high plasma velocities in the vicinity of the antenna. With that caveat in mind, we confirm that the following two panels show the obvious presence of local plasma lines (stripes in the upper and background noise in the lower panel) and gyrolines (sharp lines at $\sim 3$ and $\sim 6 \mathrm{~ms}$ to the right of the terminator). Thus, our derivation of local electron density and magnetic field magnitude is reliable for this orbit.

[43] MARSIS data from the next orbit 7971 (red dots in the upper two panels and two panels at the bottom of Figure 6) show basically the same ionospheric behavior, with a wide and well-defined ionosphere from the dayside inner edge of the magnetosheath (at 0605 UT, compare also to ASPERA3 data in Figure 5) to the termination of the AIS operations on the nightside (at 0532 UT). Again, behind the terminator (to the left), the nightside electron density is seen to decrease only slowly, which could indicate some trans-terminator plasma transport. In general, the ionosphere appears somewhat expanded along this orbit, as was also noted in the discussion of the ASPERA-3 data in Figure 5 above. The strength of draped IMF around Mars, however, has increased a little, now about $15 \mathrm{nT}$, from $800 \mathrm{~km}$ down to $500 \mathrm{~km}$ 


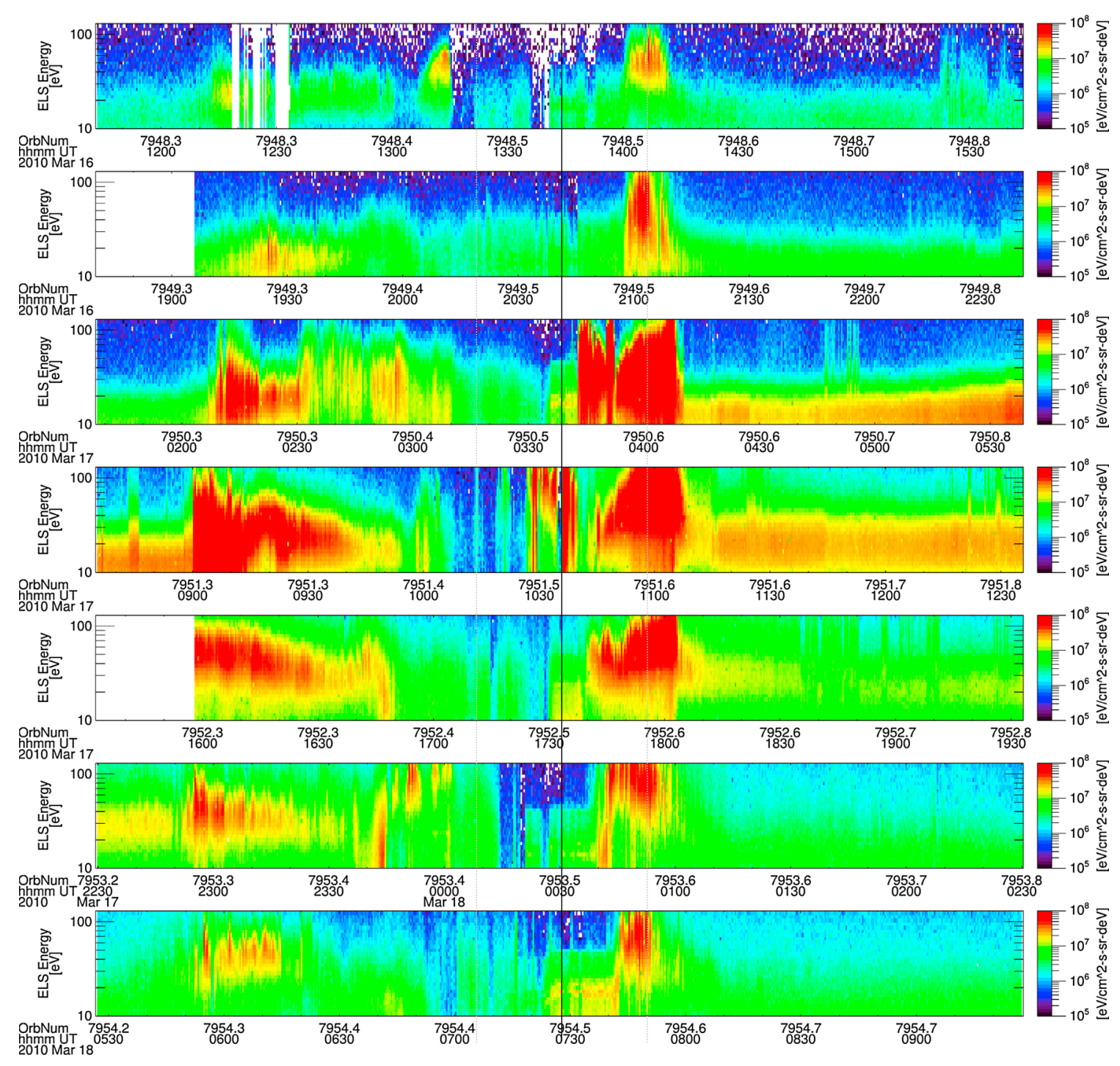

Figure 7a. ASPERA-3 electron spectra for CIR event 1, on 17 March for orbits 7948 - 7954, else as Figure 5a.

altitude, as is also well illustrated by the denser traces of gyrolines echoes in the bottom panel of Figure 6 at about 2, 5 , and $7 \mathrm{~ms}$, respectively).

[44] In summary, we conclude that during this period of quiet solar wind, the Martian ionosphere is well defined, with sharp boundaries to the adjacent magnetosheath plasma. The ionosphere appears to expand somewhat with time, as the period of solar wind quiescence continues. The strength of the dayside draped IMF is relatively constant around $12-15 \mathrm{nT}$. In fact, it appears to remain like that until the next full MARSIS AIS operation at orbit 7976 (data not shown here). Both MARSIS AIS and ASPERA-3 data indicate some transport of ionospheric plasma over the terminator, but without clear features of ionospheric plasma acceleration with altitude.

\subsection{CIR Event of 17 March 2010}

[45] As was indicated above in section 2, both the STEREO HI data shown in Figure 2 and the propagated solar wind data from near Earth orbit shown in Figure 3 indicate that a sequence of CIR-related solar wind disturbances was expected to arrive at Mars on 17 March. Typically [see, e.g., Edberg et al., 2010], the solar wind conditions during the passage of a CIR consist of a short-lived increase in the plasma density, followed by a longer period of fast but generally declining solar wind velocity. Both such features are clearly visible in the propagated WIND data and corroborated by the in situ ASPERA-3 data in Figure 3, panels d) and e). The propagated IMF during this time displays an increased variability in its absolute value, and even more variations in the radial direction (Figure 3 panel g, second panel from below).

[46] In Figures $7 \mathrm{a}-7 \mathrm{c}$, we show for this event the electron, ion, and heavy ion energy spectra, respectively, as observed during six consecutive orbits of MEX through the induced magnetosphere/ionosphere system (orbits 7948 on 16 March to 7954 on 18 March 2010). Again, we use the same format as in Figure 5, with a stacked sequence of data centered around periapsis of successive orbits. In this way, we can identify changes in the plasma populations at various locations by returning to the same magnetospheric locations from orbit to orbit along basically identical trajectories, thus monitoring spatial changes in a long-term temporal sequence. 


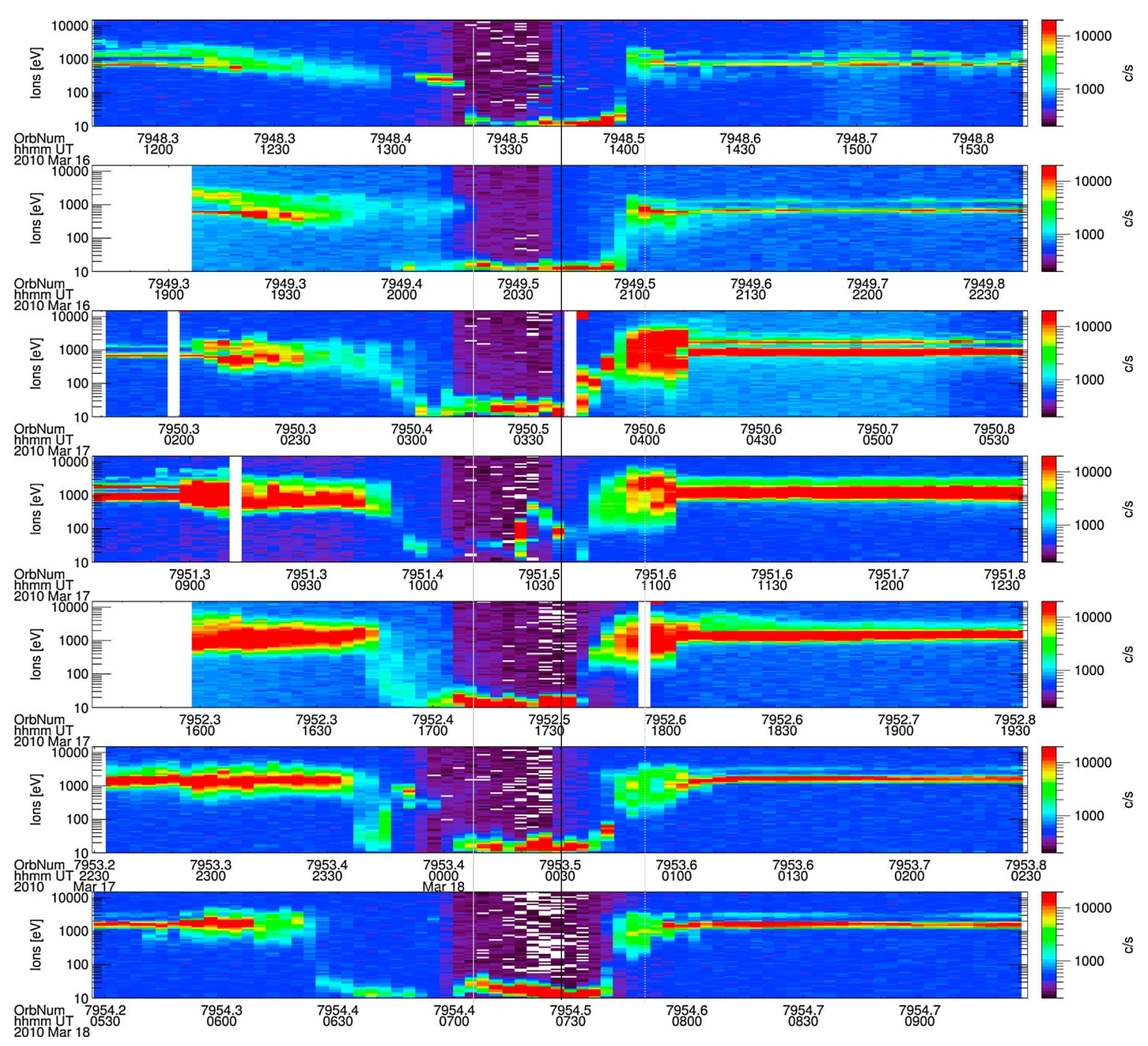

Figure 7b. ASPERA-3 all ion energy spectra for CIR event 1, 17 March 2010 for orbits 7948 - 7954, else as Figure 5b.

[47] We note immediately from Figures $7 \mathrm{a}$ and $7 \mathrm{~b}$ that the electron and ion spectra for the first (7948) and last (7954) orbit in these stacked diagrams very much resemble the undisturbed "ground state" of the Martian plasma environment, which we have discussed in section 3.1 based on Figure 5. For both these orbits (just before and just after the CIR encounter), the electron spectra (Figure 7a) indicate normal quiet time magnetosheath populations on both sides of the entry into the ionosphere, which is basically void of hot electrons. Also, the ion spectra (Figure 7b) show clearly defined and well-separated high- and low-energy regimes in the magnetosheath and ionosphere, respectively, and the heavy ion spectra (Figure 7c) show a clear separation between ionospheric and solar wind ions. A careful inspection of the full mass matrices of the ASPERA-3 data (not shown here) shows that again the few counts of apparently heavy ions in the magnetosheath and solar wind are due to proton contamination in the higher mass channels.

[48] However, right after the arrival of the CIR-related solar wind disturbance (which falls between the periapsis portions of orbits 7949 and 7950 on 17 March), the electron data during orbit 7950 suddenly display a largely increased hot magnetosheath electron population, and even some distinct electron structures within the ionospheric region of closest approach, with energies typically higher than the normal or even the enhanced magnetosheath population. On the next orbit, 7951, which is still inside the CIR encounter event, the dayside magnetosheath electron population basically remains on the same elevated level, and now even the nightside flank population has increased in both energy and density. Only on the next orbit 7952, when we see the CIR-related density increase in the solar wind decline (Figure 3, panel d), the magnetosheath electron population decreases again, finally relaxing to close to "quiet time" values by orbits 7953 and 7954, the last ones in this sequence. The prime period of CIR impact on the Martian magnetosphere ionosphere system was thus seen to last for around $20 \mathrm{~h}$, which is comparable to the duration of the CIR-related increase in solar wind density detected by MEX while outside of the bow shock (see Figure 3, panel d). The subsequent increase in the solar wind velocity (Figure 3, panel e) does not seem to have a particular strong effect on the plasma in the vicinity of Mars as seen in the data for orbits 7953 and 7054. 
OPGENOORTH ET AL.: MARS IONOSPHERIC RESPONSE

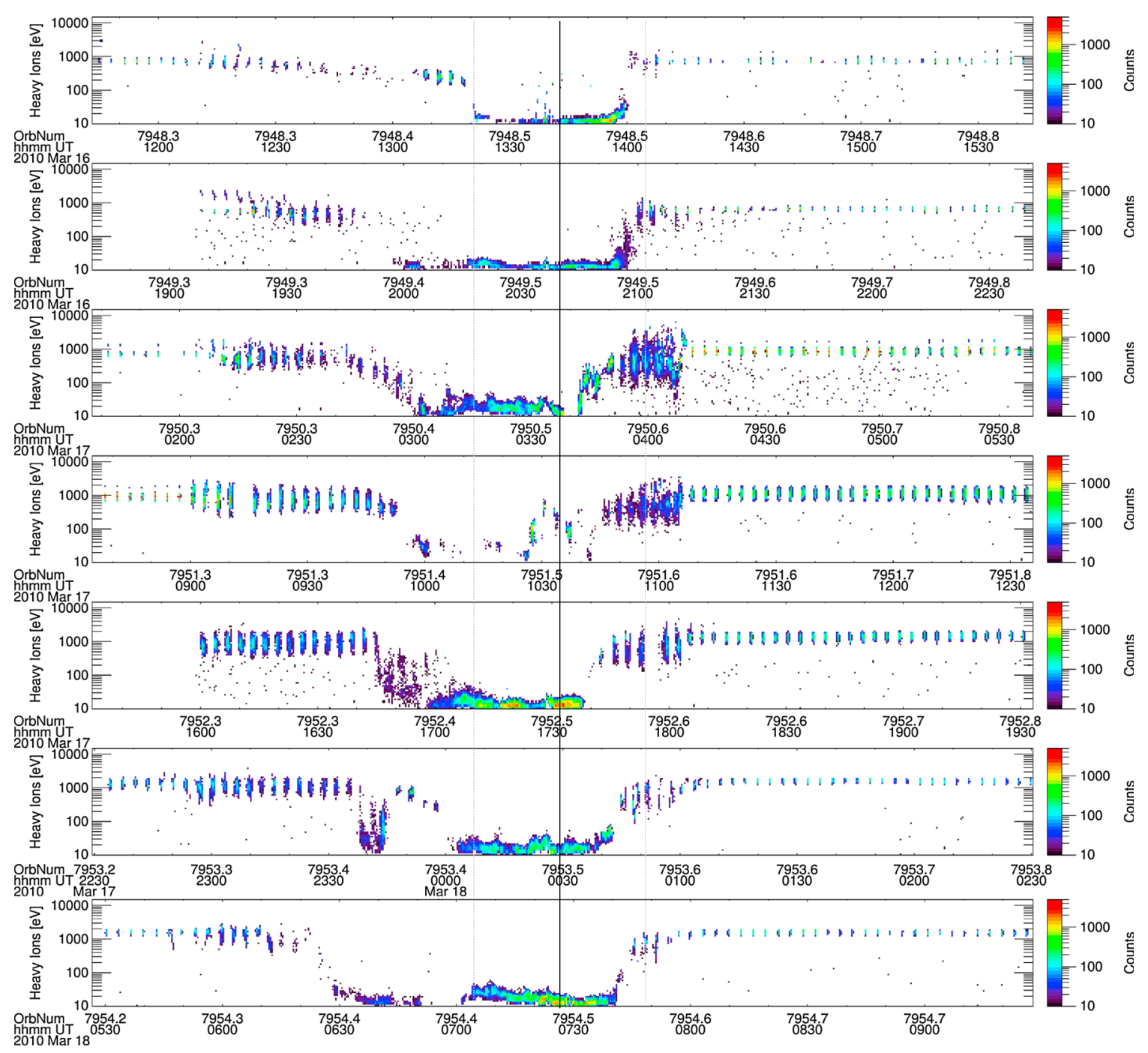

Figure 7c. ASPERA-3 heavy ion energy spectra for CIR event 1, 17 March 2010 for orbits $7948-7954$, else as Figure 5c.

[49] Inspecting the ion spectra in Figures $7 \mathrm{~b}$ (all ions) and 7c (heavy $\mathrm{O}^{+}$and $\mathrm{O}_{2}{ }^{+}$ions only), we again recognize the "boxcar" shapes of clear-cut magnetosheath-to-ionosphere boundaries in the first two and the last orbits of the sequence (7948, 7949 and 7953, 7954). In the central three orbits 7950 to 7952 , however, more gradual transitions between the hot magnetosheath ion populations and the cold ionospheric populations appear. Similar features have earlier been described by Pérez-de-Tejada et al. [2009] as signatures of increased solar wind erosion of the ionosphere in a so-called "velocity boundary layer." On the dayside, this region of accelerated ionospheric ions during orbits 7950 and 7951 is narrow, but clearly visible in comparison to the sharp transition seen, e.g., at orbit 7948 and 7949. The effects on the nightside are more complicated, and the boundary between accelerated ionospheric ions and magnetosheath ions is rather expanding, than being compressed, which would indicate a tail flaring in response to dayside compression.

[50] However, we see clearly that the accelerated ion energies are higher at larger distances from the periapsis of the orbit, which corresponds to increasingly higher altitudes, as has earlier been noted by Dubinin et al. [2006]. Figure 7c shows

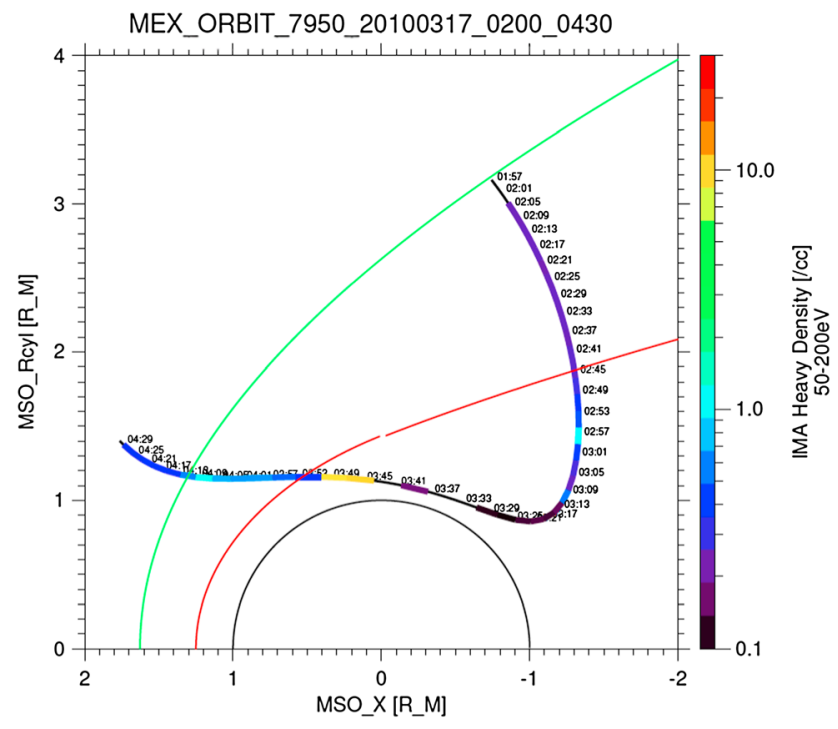

Figure 7d. Integrated local density of heavy ions in the 50-200 eV energy range in cylindrical coordinates along orbit 7950 for CIR event 1, 17 March 2010, else as Figure 5d. 
that these newly accelerated ions are indeed of ionospheric composition. Right inside the location of the MPB which was still clearly defined during the previous orbit, both on the dayside and nightside flank parts of the orbit, ionospheric ions are now seen to rise to energies of $50-300 \mathrm{eV}$. This development is very clear in orbits 7950 and 7951 , but already somewhat less pronounced during orbit 7952, when also the electron magnetosheath population had already started to decrease (c.f., Figure $7 \mathrm{a}$ above). Inspection of the individual mass matrices for these orbits indicates that the day and nightside boundaries between accelerated ionospheric ions and hot magnetosheath protons for, e.g., orbit 7950 lie at 0250 and 0350 UT, respectively, which is right at the locations where the ion energies in Figures $7 \mathrm{~b}$ and $7 \mathrm{c}$ are seen to level out toward typical sheath energies around $1 \mathrm{keV}$. These timings also confirm the dayside compression and nightside expansion (flaring) of the tail ionosphere, containing energized ionospheric plasma.

[51] Clearly during these three orbits, the location of observation of an "ionosphere proper," with heavy ion energies below $20 \mathrm{eV}$, is very much compressed on the dayside (on right side of the central periapsis portion of the figure). Following this dayside compression during orbits 7950 to 7952 , it can be noted that generally the area between the two MPB locations at orbit 7953, and even more so at orbit 7954, is much wider than any of the earlier orbits in this figure, indicating an overall relaxation of the solar wind pressure on the Martian ionosphere toward the end of the CIR passage, when Mars gets engulfed in the low-density, high-velocity stream behind the CIR shock.

[52] In addition to these general features at the boundaries, more localized or transient features can often be seen, e.g., during orbit 7951 (from $\sim 1030$ to $\sim 1040$ UT), when a narrow accelerated ion population is visible in Figure $7 b$, panel 4 . This signature occurs in concert with a similar feature in the corresponding electron spectrogram, where a population with energies above the typical solar wind and magnetosheath energies is present Figure 7a, panel 4 . These are located almost exactly at periapsis, well before the outbound dayside MPB crossing, which is crossed by MEX not before $\sim 1050$ UT. Inspecting the Cain-model for Martian crustal magnetic fields [Cain et al., 2003], we find that in this particular case the observed feature in both the electron and ion spectra is closely related to the passage of the spacecraft over a region of more intense crustal field. This magnetic field structure from below the spacecraft extends from $\sim 10: 28$ to 10:35 UT, peaking at $\sim 10: 33$ at a strength of $20 \mathrm{nT}$ at the spacecraft altitude, directly at the time of the observed structure in the particle spectra. Lillis et al. [2011] have reported similar features using MGS electron reflectometer data, attributing them to acceleration along open cusp-like crustal magnetic field lines. In this case, the field is indeed mainly radial. We will therefore ignore this feature for the purpose of this study, as it appears not to be directly related to the large-scale plasma characteristics during solar wind changes, which is the focus of this study. However, we note that increased solar wind activity, such as the passage of a CIR may considerably enhance the effectiveness of plasma acceleration processes at such crustal field magnetic cusps.

[53] Returning to the more "planetary scale" changes in the acceleration of cold ionospheric ions in the ASPERA-3 data, we want to determine in more detail where within the Martian magnetosphere/ionosphere system they are seen. We have therefore, in Figure $7 \mathrm{~d}$ for orbit 7950 , integrated the total heavy ion flux in the energy range from 50 to $200 \mathrm{eV}$ (i.e., ranging from just above typical ionospheric energies to clearly below typical magnetosheath energies) and show the total density along the orbit, again in cylindrical coordinates as in Figures 4d and 5d. Compared to the quiet time situation in Figure 5d during this CIR encounter, we now see clearly enhanced and accelerated heavy ion flux of ionospheric origin appearing right behind the dayside MPB, in the top layer of the sunlit ionosphere, just in front of the terminator, and again deeper in the magnetosphere close to the nightside flank magnetosheath. As one can easily guess from Figures $7 b$ and $7 c$, we note (without showing all the data) that for orbit 7951 and 7952 the heavy ion data look very much the same as in Figure 7d, while for orbits 7949 (before the event) and 7953 and 7954 (after the event) it looks very much like in Figure 5d, i.e., no apparent outflow of plasma within this energy range.

[54] As in the case of the quiet time magnetosphere and ionosphere in section 3.1 above, we would now like to inspect the data from consecutive orbits with MARSIS AIS data and compare it to the development of ionospheric boundaries as seen in the ASPERA-3 data in Figure 7. Unfortunately, for orbits 7951, 7953, and 7954, there are only very limited AIS data during two short time intervals per orbit (see again the green block diagram in Figure 3, panel b), which are of little use in such a study. Meanwhile full sets of AIS data were obtained for orbits 7948, 7950, and 7952 , i.e., for every other orbit in this event, which can be considered as quite fortuitous after all as they represent the situation before at the beginning and in the middle of the CIR encounter.

[55] In Figure 8a, we show (using the same format as introduced in section 3.1, Figure 6) a comparative presentation of AIS data from orbits 7948 and 7950 , i.e., right before, and at the beginning of the CIR encounter during this event (as in Figure 6 black (red) dots and central (bottom) two panels representing similar data for orbit 7948 (7950)). It becomes immediately clear that the possibility for MARSIS to derive local electron density during orbit 7948 extends about as much into the dayside high altitudes as in Figure 6, for quiet times. The local density is increasing with decreasing altitude and it is relatively evenly distributed around the terminator/ periapsis. The draped magnetic field strength is low and hardly detectable by MARSIS at about $10 \mathrm{nT}$. Again, inspection of the original data in the central two panels shows that the plasma- and gyroline signatures are significant enough to rely on the derivation of these two parameters. In contrast to Figure 6, we note that the data changed drastically for the orbit 7950, right after the CIR encounter. The local electron density decreases over the entire length of the AIS operation from dayside toward the nightside of the terminator, although at the far left side of the data in the high altitude nightside flank of the ionosphere it reaches (or even slightly rises above) the previous quiet time value. At the same time, the plasma lines (panels c and d for orbit 7948, and panels e and $\mathrm{f}$ for orbit 7950) suddenly disappear on most of the dayside portion of orbit 7950, as compared to 7948 , hence there are no local plasma density estimates possible in this particular sector (compare panel 6 to the red dots in panel 1). This region on orbit 7950 corresponds to data from 0342 UT onward, i.e., toward the dayside exit through the MPB. 


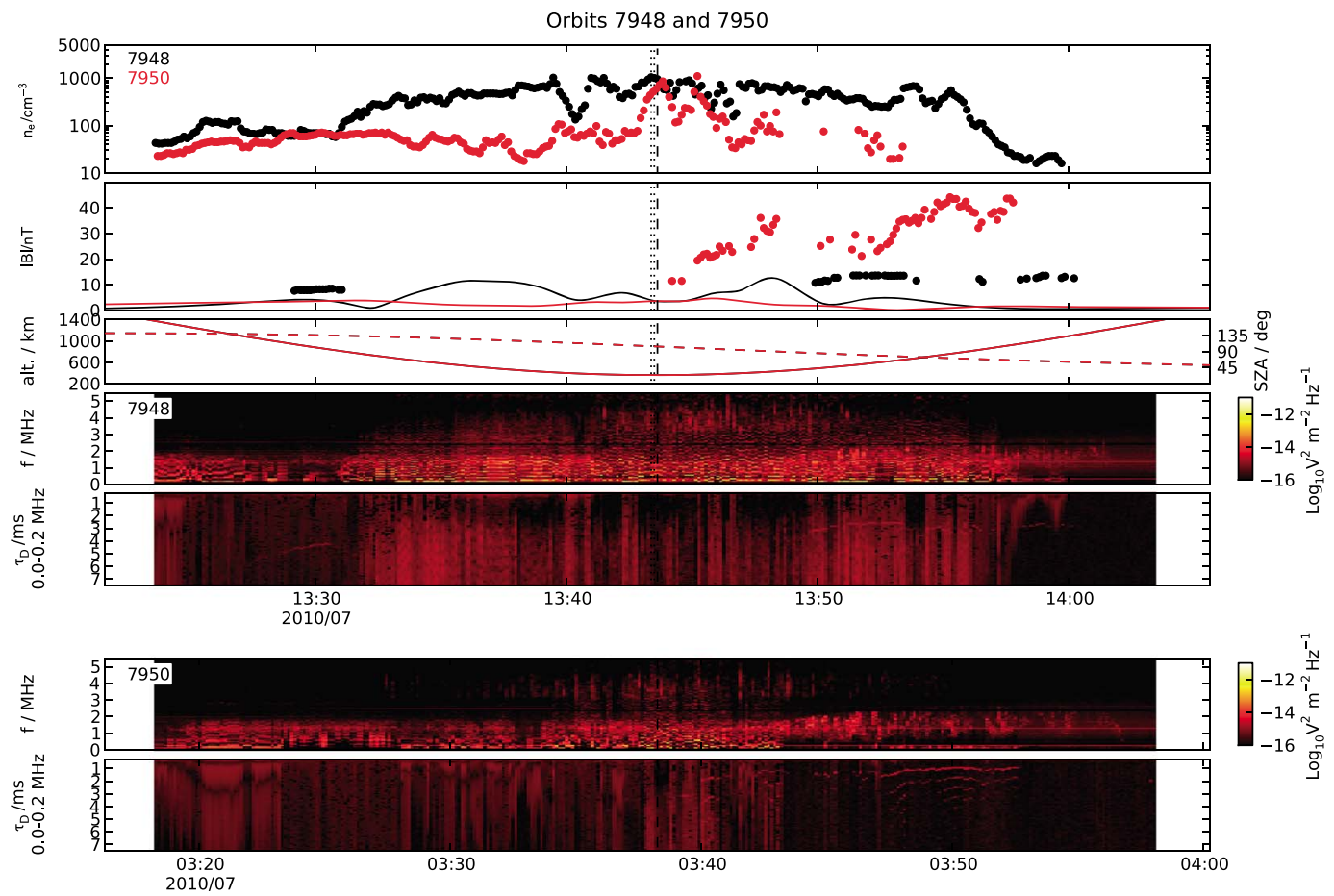

Figure 8a. MARSIS data comparison for orbits 7948 to 7950 , else as Figure 6.

Returning shortly to the ion data in Figures $7 b$ and $7 c$, this is exactly the same region in which ASPERA-3 suddenly detects suprathermal accelerated ionospheric ions during orbit 7950. Also, the magnetosheath electron population in Figure $7 \mathrm{a}$ for orbit 7950 clearly extends further inward toward the periapsis, to about 03:42 UT, in agreement with the time (i.e., location) for the loss of plasma-line echoes. It appears from this data that during the beginning of the event, when Mars encounters the strongest CIR-related peak in solar wind dynamic pressure, the entire dayside ionosphere is compressed to altitudes below the spacecraft such that MEX finds itself suddenly in an environment of low-density, but higher energy plasma, while all the dense cold ionospheric plasma seen at orbit 7948 is compressed below the spacecraft.

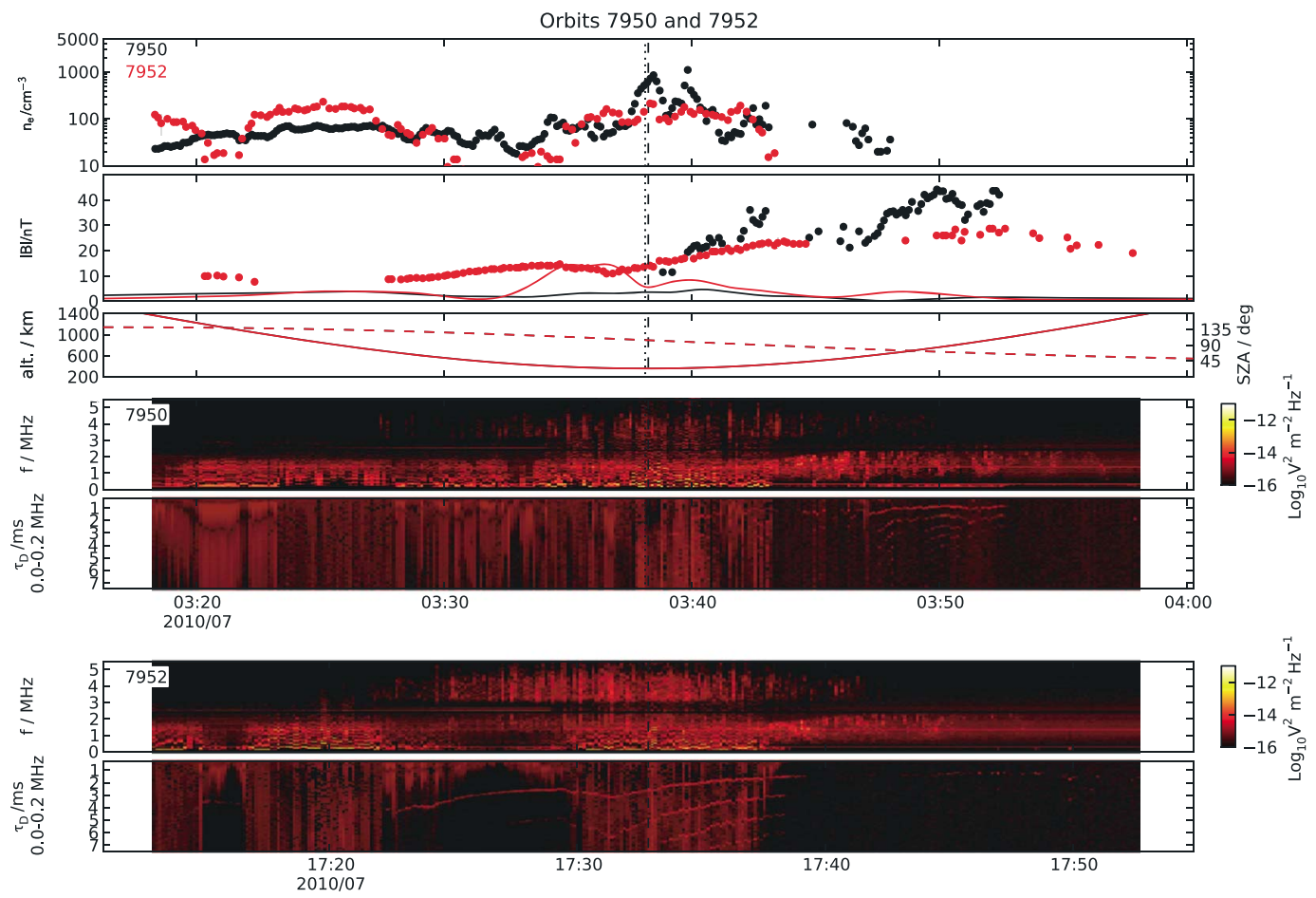

Figure 8b. MARSIS data comparison for orbits 7950 and 7952, else as Figure 6. 
[56] Another observation from Figure $8 \mathrm{a}$ is that the gyrolines become much more prominent during orbit 7950 (bottom panel), and that consequently the intensity of the draped magnetic field around the dayside portion of the planet (on the right side of Figure 8a) must have increased very much - from around $10 \mathrm{nT}$ before the event to $40 \mathrm{nT}$ at the beginning of the CIR event (compare black and red dots in Panel 2 of Figure 8a). A further close inspection of the delay data in panels $\mathrm{d}$ and $\mathrm{f}$ reveals clearly improved and much denser signatures of electron gyroline harmonics between orbits 7948 and 7950, indicating the reliability of this MEX measurement of the magnetic field. This would be in agreement with a major increase in the solar wind dynamic pressure, being the cause of the observed ionospheric compression at this time. According to Edberg et al. [2010], it would often be just in the beginning of an interplanetary CIR encounter that one would expect a drastic short-lived increase in solar wind dynamic pressure. Our propagation of solar wind data from the Wind spacecraft from Earth to Mars in Figure 3 supports this observation further.

[57] In Figure 8b, we look at the further development of the ionospheric plasma characteristics during the course of the CIR event, now by comparing data from the early and late event, i.e., for orbits 7950 and 7952 . It can be seen that the generally strong magnetic field intensity seen at orbit 7950 in Figure $8 \mathrm{a}$ (now represented by black dots in panel 2 of Figure $8 \mathrm{~b}$ ), and the compression of the dayside ionosphere, as indicated by the absence of plasma lines, prevail also during this orbit which occurs toward the end of the prime event (red dots for orbit 7952). This is in agreement with the observation of (somewhat decreased, but still prevailing) magnetosheath electrons and accelerated ionospheric ions in the same region by ASPERA-3 (see Figures 7a-7c panel 5 for orbit 7952). Inspecting the central (terminator) and left (nightside) portions of Figure $8 \mathrm{~b}$ demonstrates a further increase in the local plasma density on the nightside of the terminator, while the density at the terminator itself further decreases, as was the case already between orbits 7948 and 7950 in Figure 8a. Again, these futures are in agreement with a dayside compression (higher density ionosphere moving below the spacecraft altitude) and nightside flaring (more and denser ionospheric plasma reaching spacecraft altitude) as already discussed above for the ASPERA ion data in Figures $7 \mathrm{~b}$ and $7 \mathrm{c}$.

[58] In summary, we find that the CIR encounter leads to an overall compression of the dayside ionosphere below the spacecraft altitude. Simultaneously, we see a flaring of the nightside ionosphere, resulting in higher plasma densities at MEX orbit there during the time of the event as compared to quiet solar wind conditions before the event. On the topside of this ionosphere, compressed on the dayside and expanded (flared) on the nightside, ASPERA-3 sees accelerated ionospheric ions at energies from several $10 \mathrm{~s}$ to $100 \mathrm{~s}$ of $\mathrm{eV}$ with the energy increasing with increasing altitude. During the event, the strength of the magnetic draping is seen to increase from 10 to $40 \mathrm{nT}$ as to withstand the increased dynamic pressure of the solar wind in the shockfront of the CIR feature in the solar wind.

\subsection{CIR (and Possible CME) Event of 26-28 March 2010}

[59] During this event, the ASPERA-3 data from the solar wind (red dots in Figure 3, panels $\mathrm{d}$ and e) indicate a shortlived density increase on the afternoon of 25 March (before periapsis of orbit 7980), which is only a little earlier than the prediction of the CIR arrival time based on the propagation of Wind data from Earth to Mars. Again, the sequence of short-lived plasma-density enhancement (panel d), coinciding with a longer lasting velocity enhancement (panel d) and short-lived increase of IMF variability (panel g), indicates the arrival of a CIR at Mars. However, in this case, detailed analysis of the STEREO A SECCHI HI data also indicates the arrival of a small CME immediately after the initial CIR arrival at Mars. It appears that this CME is overtaking the CIR disturbances just about at the location of Mars within its radial expansion from the Sun. This CME solar wind structure is of course not recognizable in the propagated Wind data, as we have assumed propagation from Earth to Mars according to measured solar wind parameters at Earth, and not for fast radial CME propagation throughout a large sector of the heliosphere. We cannot safely identify any CME-related density increase from CIR features in the in situ ASPERA-3 data either, although knowledge of this particular STEREO data might help to explain some details in the ASPERA-3 observations presented below.

[60] As was done previously in section 3.2, we first show in Figure 9a a stacked series of electron energy spectra from ASPERA-3, along orbit 7979 (from 1200 UT on 25 March 2010), in the top panel, and then for seven consecutive orbits down to orbit 7986 (from 1600 UT on 27 March 2010) in the bottom panel. This data should safely cover the arrival and potential impact of the CIR- and other suspected CME-related solar wind disturbances on the Martian plasma environment.

[61] In these sequences of electron spectra for successive orbits, one can identify the beginning of the event possibly to orbit 7980, but definitively to orbit 7981, when a small enhancement of the magnetosheath population during orbit 7980 (as compared to orbit 7979) is followed by a massive compression of both the bow shock (outer edge of energetic magnetosheath electrons) and MPB (inner edge of energetic magnetosheath electrons) both on the dayside and the nightside flank of the Martian magnetosphere during orbit 7981.

[62] The count rate of the magnetosheath electrons is only a little enhanced during this compression. The compression lasts for only one orbit, and already during orbit 7982 the boundaries have moved back to where they were before the event. However, the magnetosheath electron counts and overall energies remain clearly enhanced. During the next orbit, 7983, the dayside bow shock is compressed a second time and the magnetosheath energies remain high. During orbits 7984 and 7985, the magnetosphere and magnetosheath expand again, maintaining relatively high dayside magnetosheath energies, and return to the initial ionospheric and magnetospheric ground state from before the event only at orbit 7986 .

[63] For the same sequence of orbits, we inspect in Figure $9 \mathrm{~b}$ the corresponding changes in the ASPERA-3 data throughout the event for all ion masses, and in Figure 9c for heavy ionospheric ions only. As in the electron data above, (Figure 9a) orbit 7979 clearly represents the quiet ground state of the magnetosheath and the magnetosphere (compare also to the top panel of Figure $7 \mathrm{~b}$ and entire Figure $5 \mathrm{~b}$ ). The start of the event at orbit 7980 is better seen in this ion data, with an initial increase of both magnetosheath ion counts and energies. We also note an immediate start of energization of cold ionospheric ions toward the nightside MPB. 


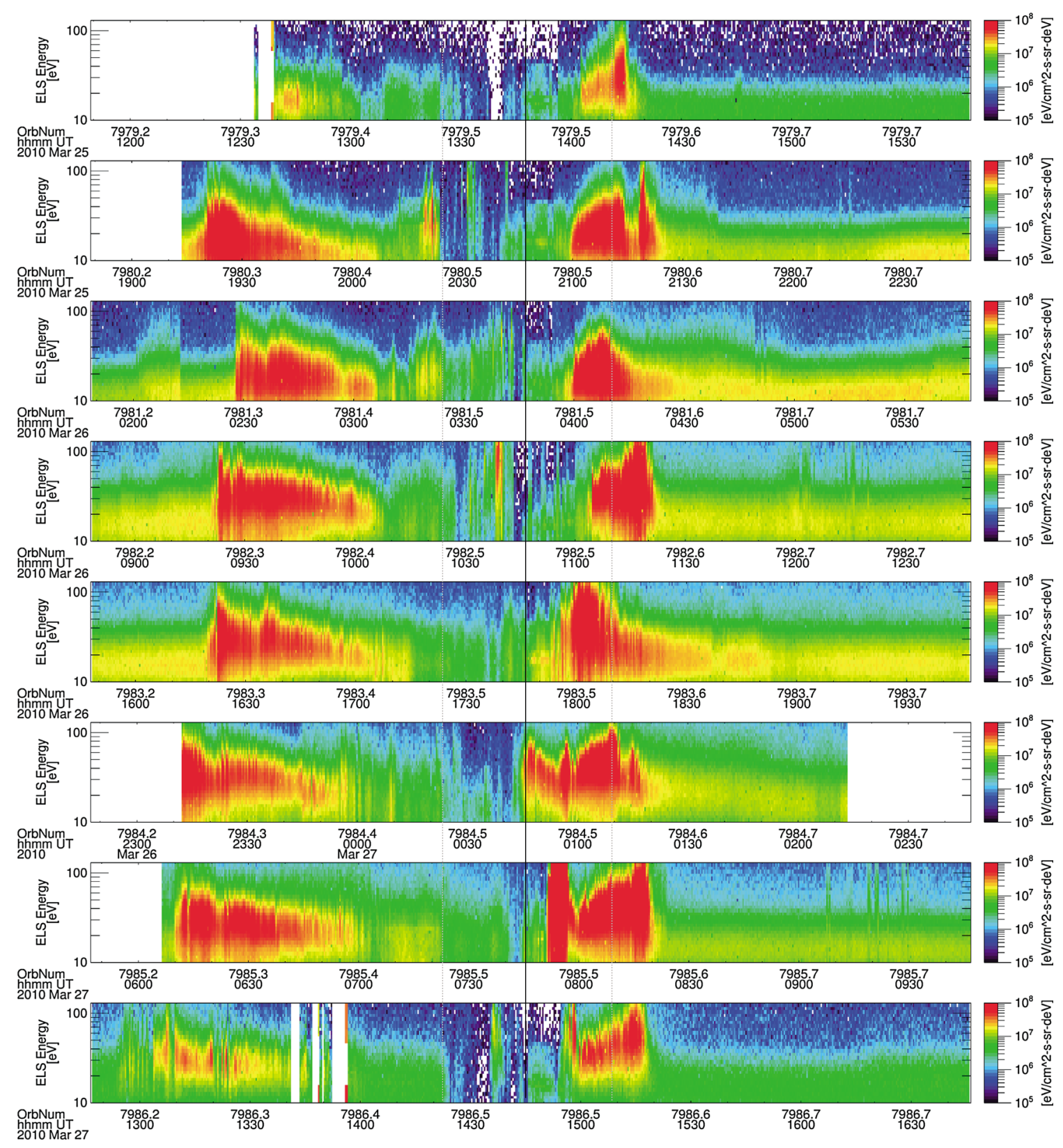

Figure 9a. ASPERA-3 electron spectra for orbits 7979 to 7986, CIR and CME event 2, else as Figure 5a.

During orbit 7981, the magnetospheric compression and the magnetosheath temperature (energy spread) and density (count rate) enhancement become more pronounced than in orbit 7980. Furthermore, the sloping energy distributions around both MPB locations on the day and nightside, with higher energies toward higher altitudes, indicate a more effective acceleration of cold ionospheric ions. In orbit 7981, we even see some signatures of ion acceleration above the central ionospheric parts of the orbit, which in this case are not related to crustal anomalies below the spacecraft. During orbit 7982, the separation in energy between magnetosheath and ionosphere becomes sharp again, and an overall expansion of the dayside MPB and bow shock is clearly visible, as was already noted in the corresponding electron data for this orbit in Figure 9a. In orbit 7983, the entire magnetosphere ionosphere system is compressed a second time, and again there are more pronounced slopes in the energy distribution with altitude at both the day and nightside boundaries between magnetosheath and ionosphere, indicating acceleration of ionospheric ions. This renewed feature of ionospheric ion acceleration enhances subsequently during orbits 7984 and 7985, and returns again to a more quiet-time boxcar shape at orbit 7986 . We note that this event, which we suspect to be a CME following a more typical CIR front, displays the most pronounced dayside ion acceleration (or topside ionospheric plasma erosion), seen in this study. This effect may be connected to the special plasma parameters (longer lasting blob of high-velocity high-density plasma) within a CME compared to the short density shock of a CIR. Again, we have inspected that the individual mass matrices for these data and the boundaries between heavy ionospheric ions and apparent heavy ion 


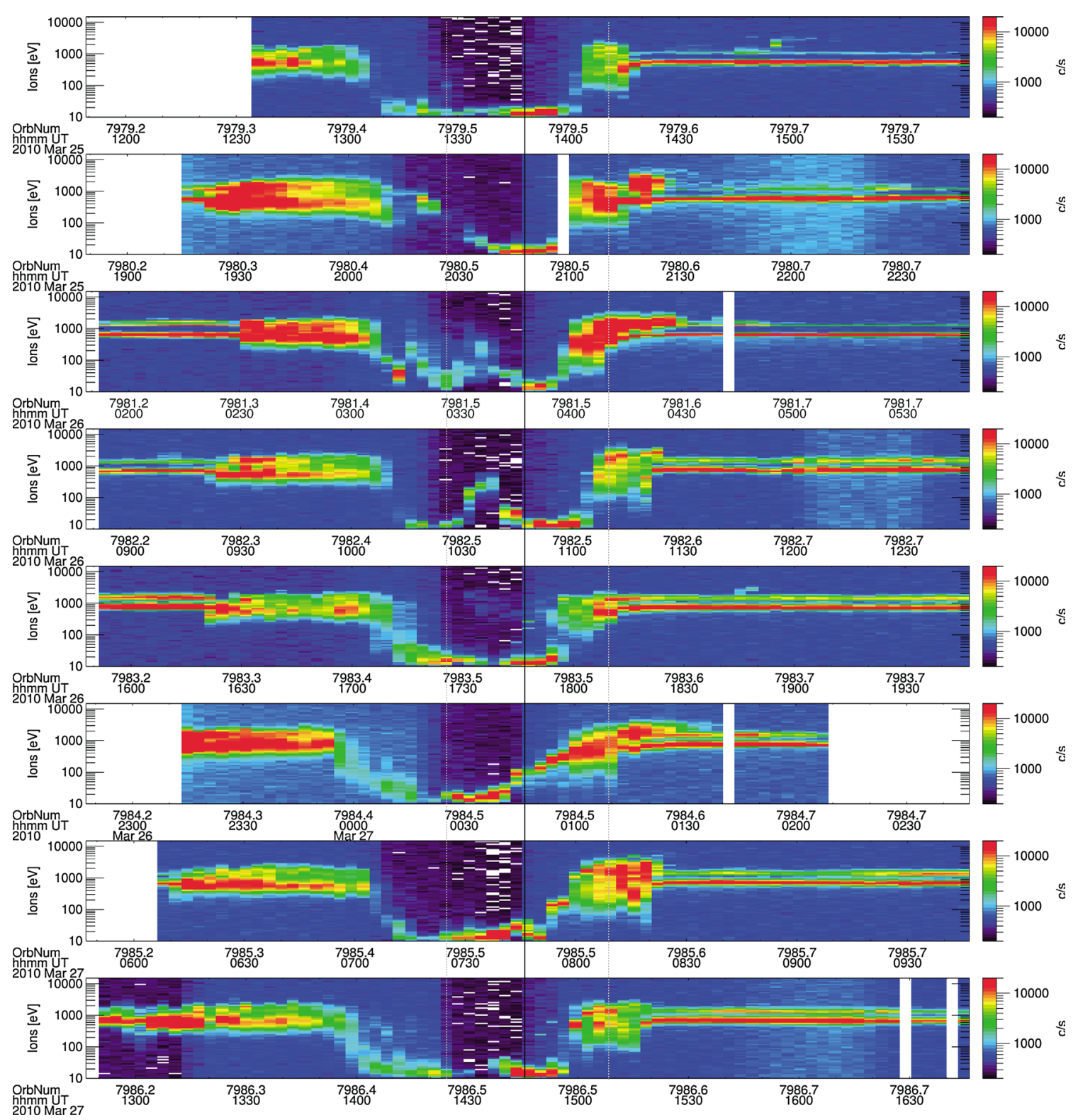

Figure 9b. ASPERA-3 all ion energy spectra for orbits 7979 to 7986, CIR and CME event 2, else as Figure 5b.

counts in the magnetosheath are located where the energy of heavy ions is seen to level out toward just below $1 \mathrm{keV}$ in Figure 9c, both on the day- and nightside of the orbit.

[64] We note again that during orbits 7980, 7981, and most pronounced during orbit 7982 , there are energetic electron and ion structures within the nominal cold ionosphere close to periapsis, which this time are not clearly related to the passage of the spacecraft close to magnetic anomalies. They could thus be related to intrusion of the hot magnetosheath or plasma sheet plasma to spacecraft altitudes during the extreme compression of the upper ionosphere. Below we will inspect the MARSIS data for this event in detail to understand more about this feature.

[65] For the central portion of this event, we have only two - but fortunately consecutive - orbits with full MARSIS AIS operation - orbits 7982 and 7983 - which happen to be just before and during the second compression of the BS and
MPB during this event. There are also full MARSIS AIS data available from the periapsis portion of orbit 7978 , i.e., well before the event. We note that orbit 7978 has a very similar structure of the electron and ion data (not shown here) as orbit 7979 in Figure 9, characteristic of the undisturbed solar wind.

[66] First, we compare in Figure 10a the data from before the event (7978) with the first available orbit in the event, which is right between the two magnetospheric compressions (orbit 7982). We use the same format as introduced in Figures 6 and 8 above. In the top panel, we see that the local electron density on the dayside of the terminator was very similar before and in the middle of the event, i.e., between the two compressions. A corresponding similarity is also visible around the dayside MPB in the electron and ion data from orbit 7979 and 7982 in Figure 9. We have noted above that during orbit 7982 at 1030 to 1045 UT, which is just behind (or to the left of) the terminator, there is a strong very localized 


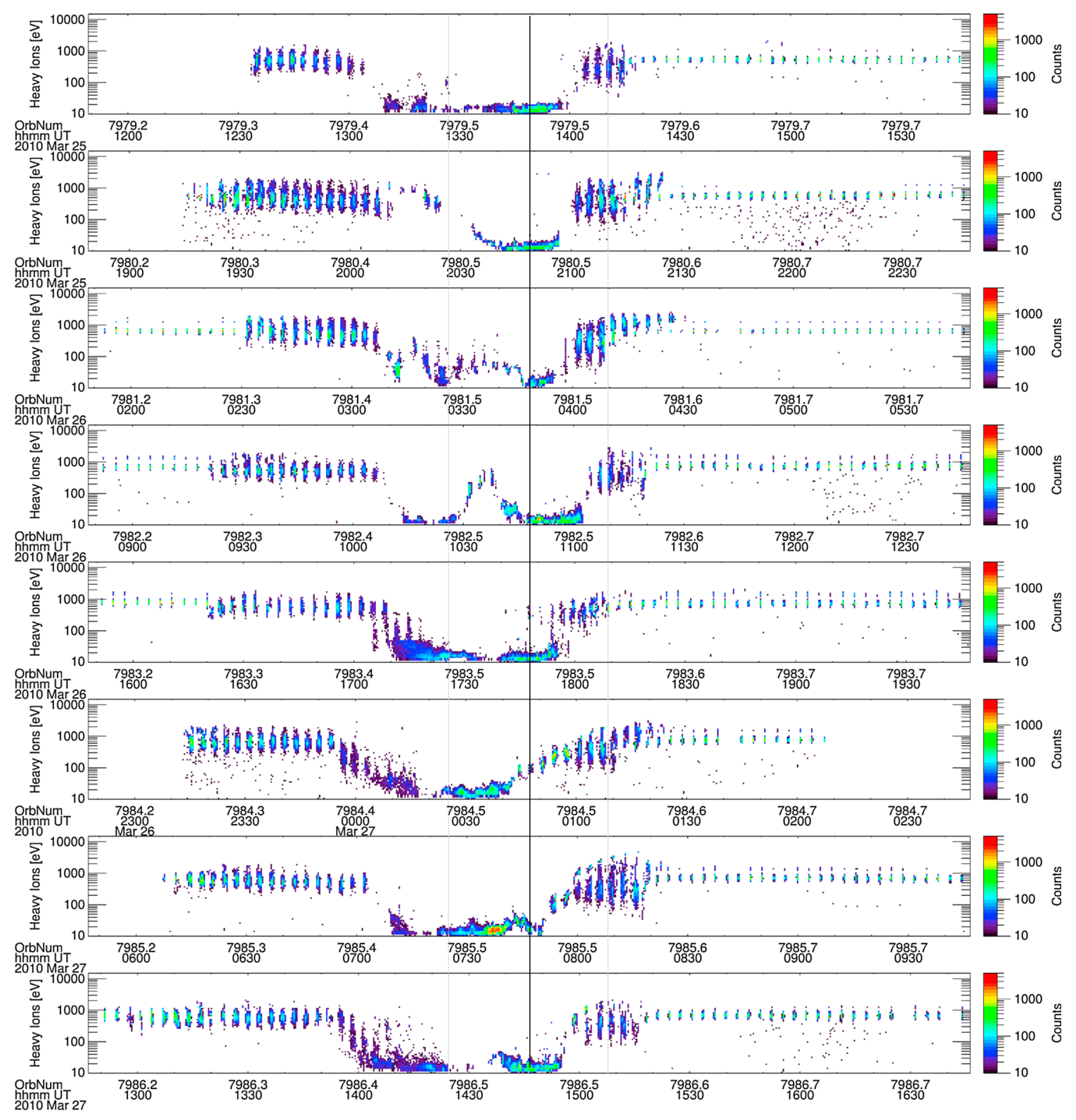

Figure 9c. ASPERA-3 heavy ion energy spectra for orbits 7979 to 7986, CIR and CME event 2, else as Figure 5c.

signature of accelerated electrons and ions, which could be related to the intrusion of magnetosheath plasma to the spacecraft altitude at this time of considerable solar wind activity. In the corresponding AIS data in Figure 10 (red dots and lower two panels), not unexpectedly both plasma and gyrolines disappear at this time (from 1030 to 1048 UT at orbit 7982), which is just as long as the suspected magnetosheath or magnetosheath plasma intrusion is seen in ASPERA-3 data. Consequently, the local ionospheric electron density is seen to be low or not detectable at all. Furthermore, we note from Figure 10, second panel from above, that the dayside draped magnetic field strength is 10 nT during orbit 7978 , but clearly enhanced to above $20 \mathrm{nT}$ during orbit 7982, within the event, again indicating higher solar wind dynamic pressure.

[67] Next, in Figure 10b, we compare MARSIS AIS data from orbit 7982 and 7983 , i.e., for the second large-scale magnetospheric compression during this event, as identified from the ASPERA-3 electron and ion data in Figure 9. Again, we see that before this compression event (black dots from orbit 7982), there is clearly a much wider dayside ionosphere than when ASPERA sees the strong compression of the MPB (red dots for orbit 7983). As discussed above in section 3.2 , this is induced from the sudden disappearance of plasma lines from the dayside (right portion of data) toward the terminator. Also, wherever local electron density can be derived (closer toward the terminator (periapsis), but still on the dayside (right) portion of the orbit) it is clearly lower after the compression event than before. On the other hand, the data from orbit 7983 show that there is now clearly more electron density on the nightside of the terminator, even if the short event of intrusion of energetic plasma to spacecraft altitudes during orbit 7982 does not allow such a comparison everywhere on the nightside. Nevertheless, the 


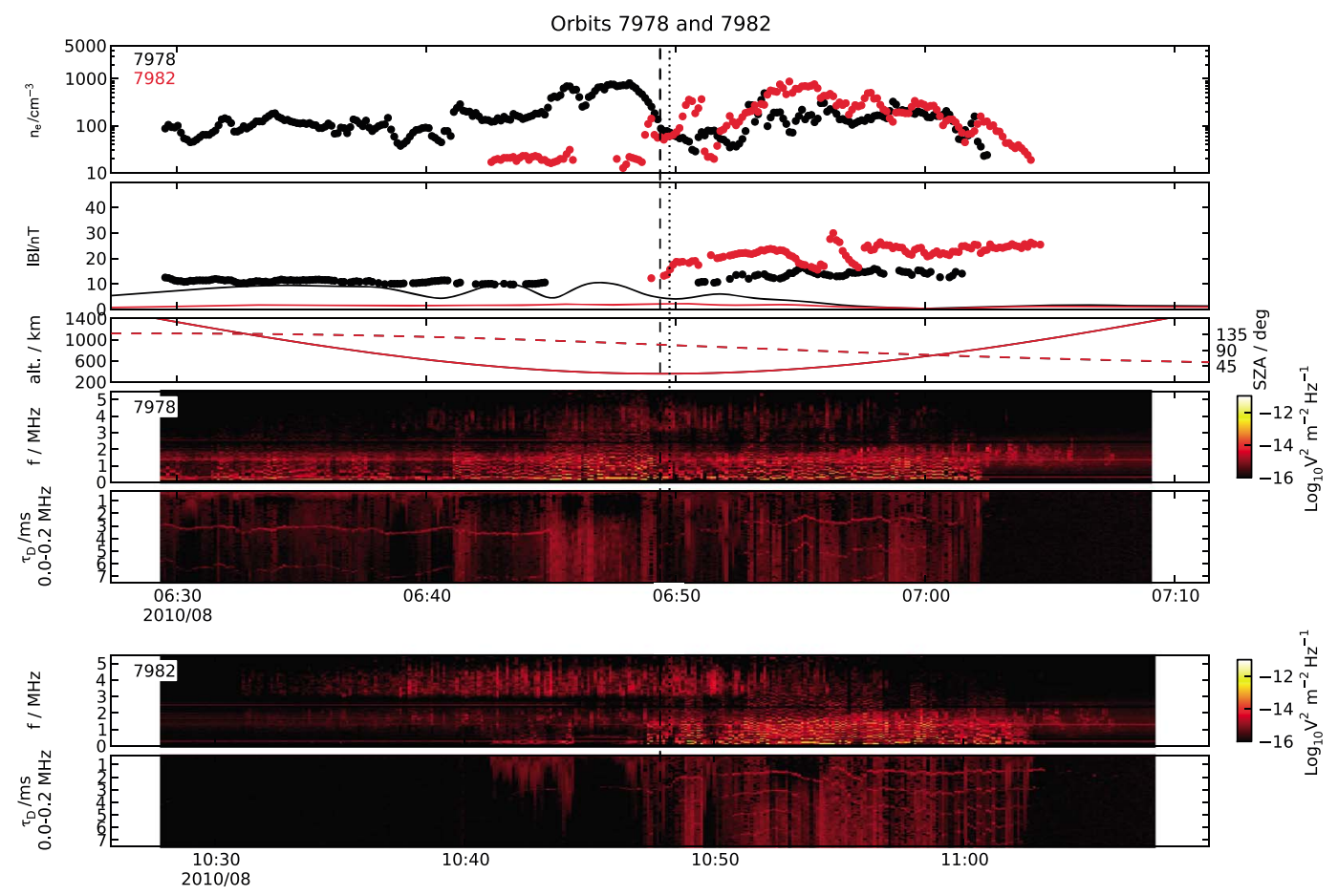

Figure 10a. MARSIS data comparison for orbits 7978-7982, else as Figure 6.

local electron data just before and after the intrusion event on orbit 7982 are clear enough to confirm a similarity in the ionospheric behavior to what we already have identified in Figure 8a between orbits 7948 and 7950 (and even between 7950 and 7952), namely a compression of the ionosphere (lower density) on the dayside and higher density on the nightside ionosphere during compression events. In the bottom panels of Figure 10b, it is also very striking to see that when the plasma lines disappear on the dayside of the periapsis, they suddenly appear much more pronounced behind the periapsis location, which means that the density estimates there are very reliable in this case. In contrast to Figure 8 , we cannot identify clear changes in the intensity of the draped magnetic field during this event. It is moderately strong (20 nT) during orbit 7982, before the compression, while subsequently during orbit 7983 MARSIS sees

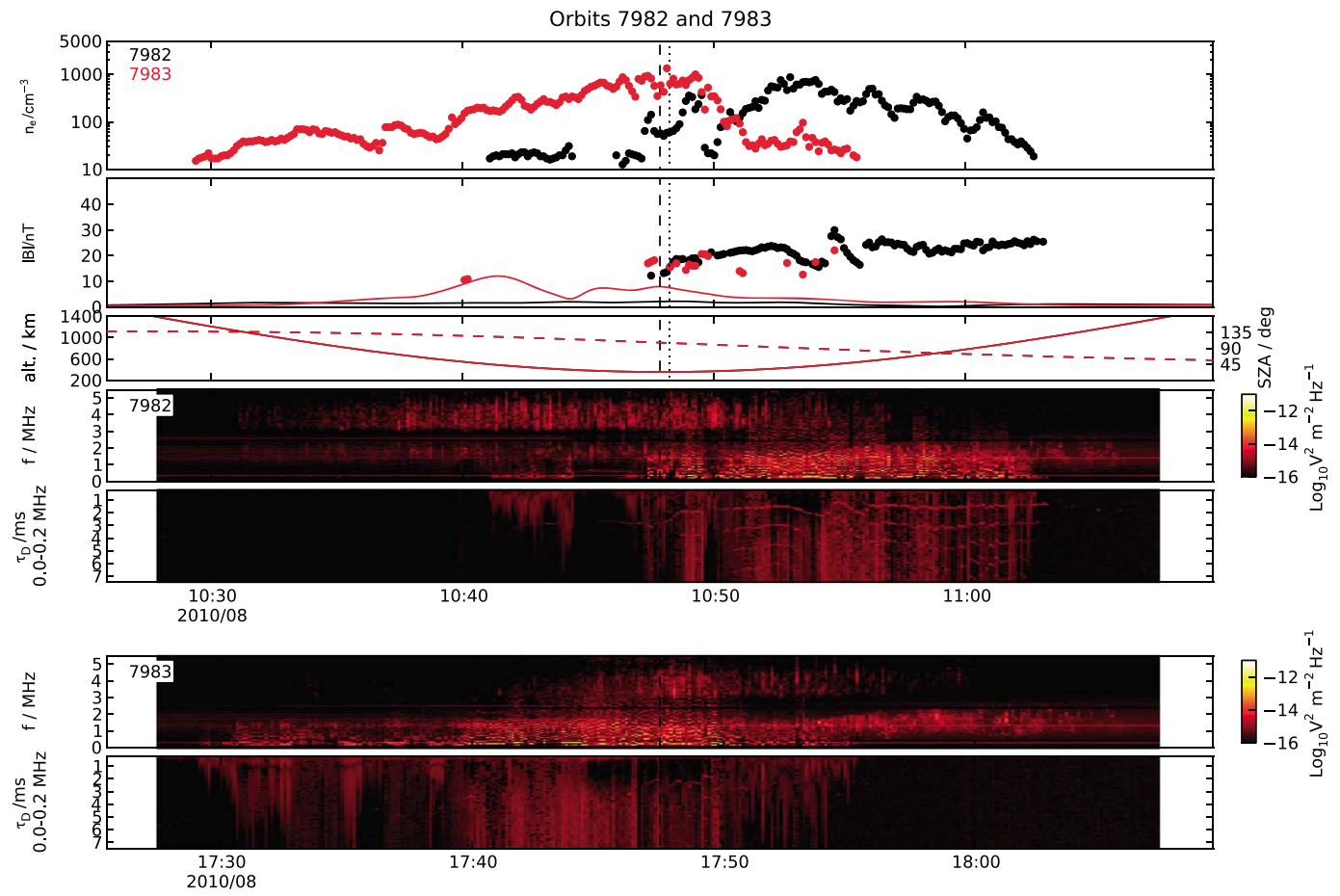

Figure 10b. (b) MARSIS data comparison for orbits 7982-7983, else as Figure 6. 


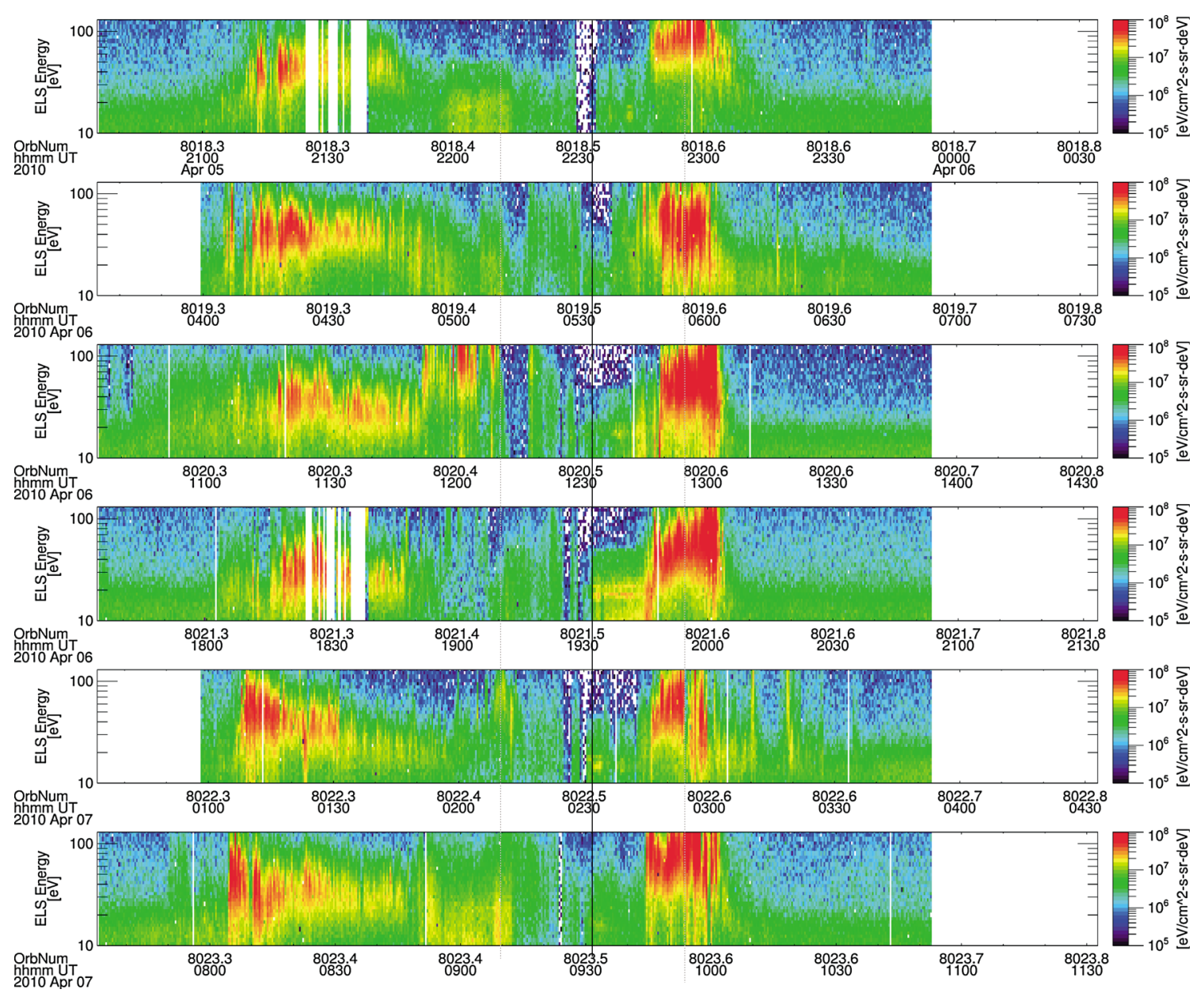

Figure 11a. ASPERA-3 electron energy spectra for orbits 8018-8023, CME event 6 April, else as Figure 5a.

neither gyrolines nor any other appreciable radio signal below $0.2 \mathrm{MHz}$, likely due to the extreme compression of the ionosphere below the spacecraft altitude, suggesting that MEX remains in the magnetosheath throughout the inbound part of the orbit. Thus, stronger magnetic field may indeed exist, but cannot be measured with the gyroline technique in this state of ionospheric compression. With the MARSIS antenna exposed to the fast flowing magnetosheath plasma, it is very likely that no radio signals from plasma instabilities, which are created in the local plasma by the emission of radio waves from the antenna, will ever return to the receiver, explaining the complete black-out of local radio noise in this channel (compare to the extreme intrusion of the solar wind ions to these altitudes in Figures 9b and 9c).

[68] We will return to these observations below in the discussion section, but it is obvious that these observations on the dayside imply a change in both the altitude and shape of the MPB, rather than a bulk transport of plasma away from the dayside. The ionosphere must still be present below the $\mathrm{s} / \mathrm{c}$ altitude. If the MPB gets compressed on the dayside below spacecraft altitude and flares out to higher altitudes on the nightside, one should expect plasma lines and gyrolines to disappear in the AIS data, because of the antenna being suddenly imbedded in fast flowing magnetosheath plasma, therefore preventing the detection of these signals. The sudden appearance of such features on the nightside must, on the other hand, mean that the plasma density there has suddenly been increased. Thus, some ionospheric plasma from the compressed dayside ionosphere must have diffused either from below or become pressed over toward the nightside, as there is no local ionization source at these altitudes.

\subsection{CME Event of 6 April 2010}

[69] The last event, which we chose to discuss in this overview, is associated with the only major CME that occurred throughout the Earth-Mars conjunction campaign in 2010. We are aware of the fact that this event maybe suboptimal, as it may have affected Mars only partially, with the flank portion of the main CME plasma cloud. However, in this overview paper, we wanted to cover the complete envelope of possible solar wind variability, and this was the best, and also already best studied CME in the campaign period (see below). It originated from the Sun in an approximately Earth-bound direction on 3 April 2010 and reached Earth in the morning of 5 April, where it created the first major space weather event of this solar cycle. The consequences of this $\mathrm{CME}$ in the Earth magnetosphere were most probably responsible for the loss of the Canadian telecommunication satellite Galaxy 15 [Allen, 2010]. The initial development and propagation of this CME in the inner heliosphere toward $1 \mathrm{AU}$ have been modeled in great detail by Rouillard et al. [2010], using the ENLIL model for solar wind propagation 


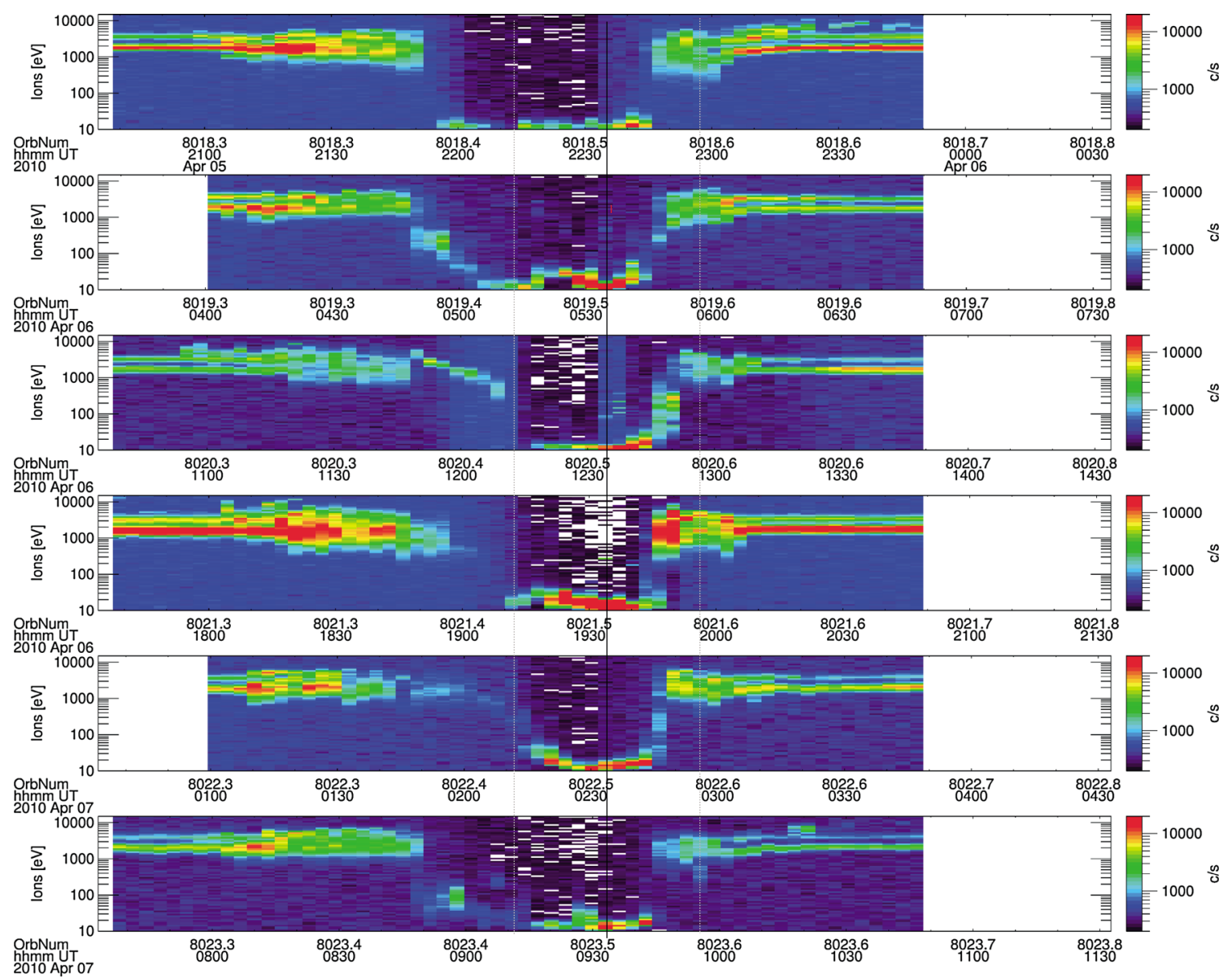

Figure 11b. ASPERA-3 all ion energy spectra for orbits 8018-8023, CME event 6 April 2010, else as Figure $5 b$.

in the heliosphere [Odstrcil and Pizzo, 1999, 2009]. Extrapolating from the known expansion of this CME toward Earth (Figure $6 \mathrm{f}$ of Rouillard et al. [2010]), one can make the prediction that the left flank of the CME (which is on the downstream side in the sense of the Parker spiral solar wind expansion) should have arrived at Mars on 6 April. Because of the late occurrence in the campaign, this unfortunately means that Earth and Mars have already been separated very far from radial conjunction in the solar wind. Thus, if the central main body of the radially expanding CME cloud did hit Earth, then consequently Mars will only be affected by the outer left (leading) edge of the expanding CME front, when looking from Mars toward the Sun.

[70] Again, starting with the ASPERA-3 electron data, we show in Figure 11a electron spectrograms from orbits 8018 to 8023 , covering late 5 April to early 7 April 2010, which with good margin includes the expected arrival time of this CME at Mars on 6 April 2010. Indeed, in this data the CME arrival at Mars can readily be identified by an enhancement of the dayside magnetosheath electron density and a compression of the nightside bow shock (left portion of Figure 11a) during orbits 8020 and 8021 . Some enhancement in the dayside magnetosheath electron density starts probably already during orbit 8019 , but is not very pronounced in the electron data until orbit 8020 . It should be noted, however, that during this entire event, and even during the quiet time before the event the initial energies of magnetosheath electrons are considerably higher than during any of the previously discussed events in this paper, which indicates some already ongoing background disturbance of the Solar wind before the CME arrives at Mars. This is also clear from the propagated and in situ measurements from the solar wind around Mars in Figure 3. A period of long-lived increase in Solar wind velocity and density starts already as early as 1 April. The resulting increase in the overall dynamic pressure between 1 April and 6 April is actually of the order of the enhancements discussed in the previous two events. This is mainly caused by an overall increase of the solar wind velocity from below $400 \mathrm{~km} \mathrm{~s}^{-1}$ in March to close to $600 \mathrm{~km} \mathrm{~s}^{-1}$ in this period in early April. The red blocks in the third panel of Figure 3 indicate that there are several small events identified from Mars Express data during this time, which we chose not to include in this paper.

[71] The short-lived intense electron density and velocity spike at midday on 4 April correspond to the propagated data from the CME arrival at Earth, as seen by Wind. However, in this case the standard estimation of the arrival time at Mars is completely wrong and misleading, as fast CMEs expand more or less radially in the heliosphere, overtaking all other solar wind structures in their way, thereby heavily disturbing the normal Parker spiral structure [see Rouillard et al., 2010].

[72] The actual true arrival time of the CME-related features at Mars can best be seen in the morning of 6 April in 


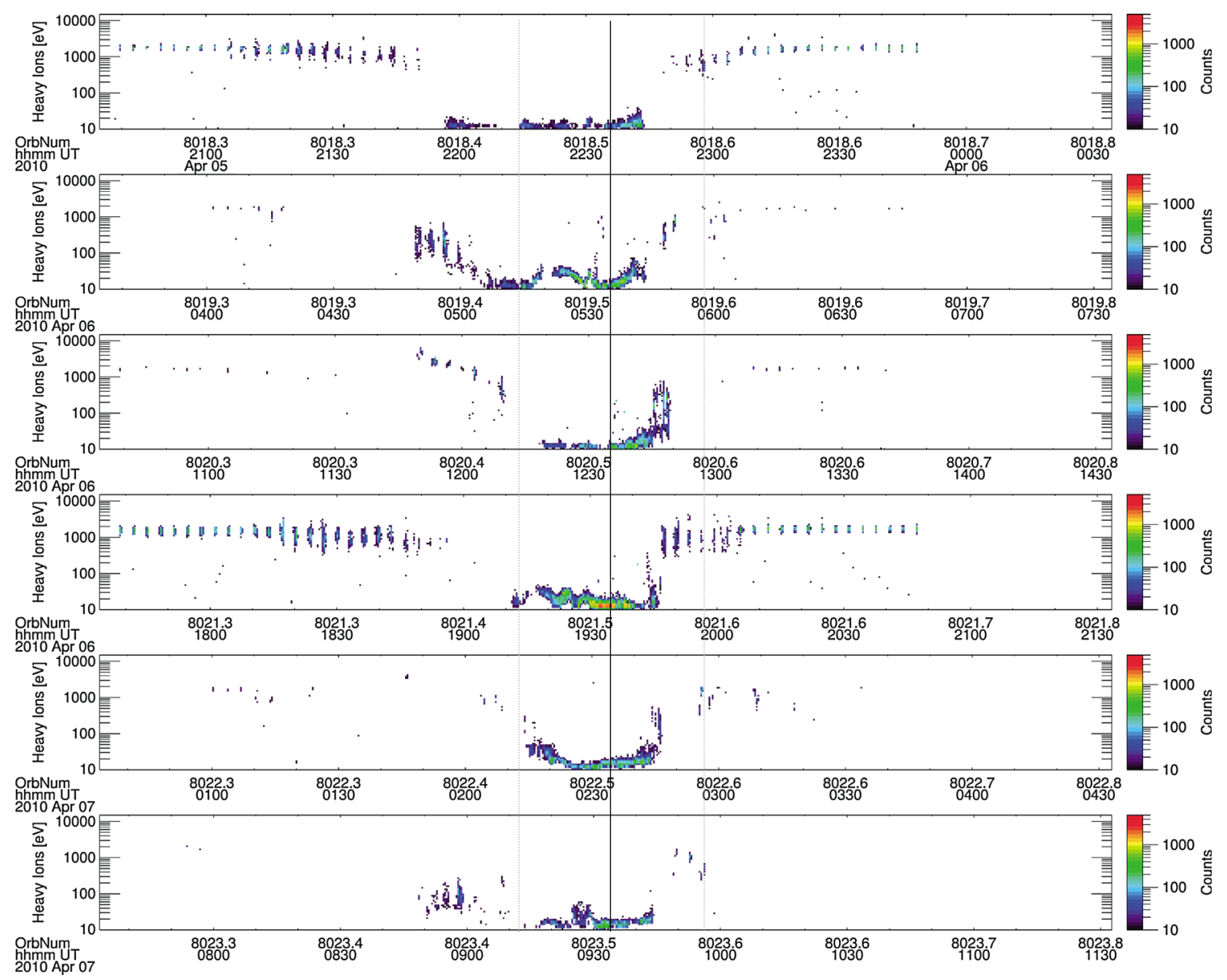

Figure 11c. ASPERA-3 heavy ion spectra for orbits 8018-8023, CME event 6 April 2010, else as Figure 5c.

Figure 3, in the ASPERA in situ data (red dots), when there is a sudden increase of solar wind velocity over already high values. This event is accompanied by only a minor increase in the solar wind density, nevertheless leading to a doubling of the solar wind pressure at Mars early on 6 April. The relatively weak signature in the electron density data supports our suspicion that the central CME ejecta do not fully engulf Mars during this event, but pass between the heliospheric longitude of Earth and Mars.

[73] In Figures 11b and 11c (again using the same format as in Figures 5, 7, and 9), we show the corresponding ion data for all (b) and heavy ions only (c) from the ASPERA IMA experiment. Also, these data exhibit the same general behavior as already observed during the previous events. Starting out from a quiet time situation, with "boxcar"-shaped well-defined boundaries between high-energy (light) magnetosheath ions and low-energy (heavy) ionospheric ions at orbit 8018 on 5 April, the arrival of the CME is indicated by a sudden acceleration of heavy ionospheric ions during orbit 8019, mainly on the nightside portion of the ionosphere. Again, heavy ionospheric ions are accelerated to energies of several $100 \mathrm{eV}$, increasing with altitude. During orbits 8020 and 8021, this acceleration rises to higher and higher energies, reaching eventually typical background magnetosheath energies of solar wind particles up to $1 \mathrm{keV}$. On the nightside (left) portion of orbits 8020 to 8022 , this acceleration also clearly extends further and further into a region of the orbit, which earlier (during orbit 8018 and 8019 ) has been dominated by cold ionospheric ions. Only toward the end of the solar wind event at orbit 8023, the shape of the boundaries between the ionospheric cold and magnetosheath hot ions relaxes to the ordinary boxcar shape with clearly defined boundaries, which we identified above to be characteristic for quiet solar wind conditions.

[74] From Figure 11c, it becomes clear that the ions responsible for the sloping energy spectra close to the MPB locations are of ionospheric origin, and are not at all present before the event (orbit 8018, top panel) and much less accelerated after the event (orbit 8023, bottom panel). This event appears to be somewhat longer lasting than the previous events as we see effects of ion acceleration during four full orbits $(28 \mathrm{~h})$ This is, however, in good agreement with the increase of Solar wind pressure and velocity seen by ASPERA-3, when outside the Martian bow shock (see discussion of Figure 3 above). The previous two events were caused by considerably shorter spikes in the solar wind electron density, and consequently the duration of the deformation of plasma boundaries and ion acceleration was also seen to be much shorter then.

[75] Moving on to the MARSIS AIS data from this event, we note that because it occurred very late in the campaign, the coverage of AIS data is, in fact, worse than during the main body of the campaign, as becomes clear from the scarcity of full green blocks in the second panel of Figure 3. Nevertheless, we were lucky to have at least two consecutive 

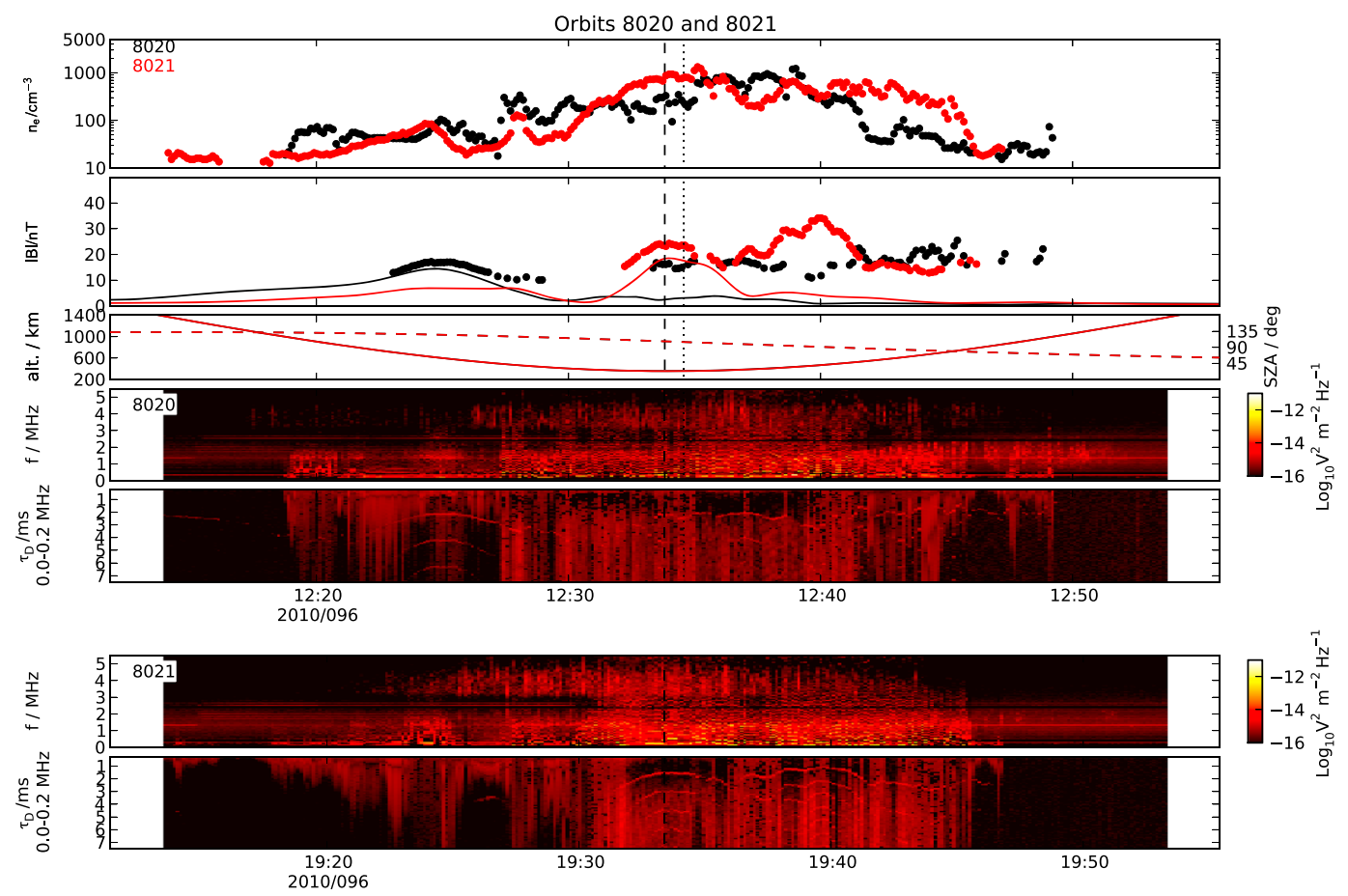

Figure 12. MARSIS data comparisons for orbits 8020 and 8021, else as Figure 6.

full periapsis operations of MARSIS AIS in the middle of the event (from orbits 8020 and 8021). Carefully inspecting the ASPERA-3 ion data in Figures $11 \mathrm{~b}$ and $11 \mathrm{c}$ shows that there is a clear intensification in the magnetosheath plasma between orbit 8020 and 8021, associated with a clearly increased cold ionospheric plasma density on both sides of the terminator, which is crossed at about 1935 UT during orbit 8021. Also, the dayside ionosphere is clearly compressed between orbits 8020 and 8021 .

[76] In Figure 12, we present the MARSIS data from these two orbits in the same format as in Figures 6, 8, and 10 above. The most prominent difference between these two orbits is the drastic increase in the intensity of the IMF draping from 10-20 nT during orbit 8020 , to $20-30 \mathrm{nT}$ during orbit 8021 , indicating increased solar wind dynamic pressure. Also, in further agreement with the ASPERA data, the ionospheric electron density is overall larger and wider in latitudinal coverage during the later orbit. A compression of the dayside ionosphere can only be seen by the disappearance of a few relatively low-density estimates on the far right side of the top panel, but, nevertheless, they confirm the clearer ASPERA signatures of a dayside compression. On the other hand, as in the other cases discussed above, there is an overall increase in nightside local electron density at spacecraft altitude, while the dayside values close to the terminator are somewhat reduced during the compression event.

[77] In summary, we note that also for the arrival of CMErelated solar wind pressure changes the Martian ionosphere gets compressed on the dayside, with clearly elevated signatures of heavy ion acceleration, particularly on the nightside, again with increasing energy for increasing altitudes. It is interesting in this context, that in spite of the increased background disturbance level, and the fact that this CME hits Mars only partially, it still stands out as a remarkable event in both the ASPERA and MARSIS data. The CME associated features last for almost $30 \mathrm{~h}$, throughout four consecutive orbits, while the CME event engulfs Mars. The signatures of magnetospheric compression are again accompanied by increased density of draped IMF on the dayside.

\section{Discussion}

[78] Above, we have presented electron and ion observations made by the Mars Express ASPERA-3 instrument for selected intervals during a period of one month in the spring of 2010. This period was selected for a dedicated campaign as Mars was located downstream of Earth in the solar wind, a constellation which occurs about every two years. For this campaign, the usual rate at which the MARSIS instrument is operated in AIS mode was substantially increased, through constructive co-operation within the Mars Express instrument teams in the ESA Science Operation Working Group. In this way, we could achieve several consecutive (or at least regularly repetitive) full periapsis operations of the topside sounder, allowing us to compare the dynamics in the boundaries and acceleration processes seen in the ASPERA-3 data for energetic and suprathermal $(>10 \mathrm{eV})$ electrons and ions with the behavior of the colder ionospheric plasma as seen by MARSIS AIS at (and even below) the spacecraft altitude.

[79] Due to the very late start of the present solar cycle, the conditions during this campaign were very much characteristic of solar minimum, with only a few clear CIRs and even fewer CMEs occurring. Thus, our conclusions must be seen in the light of such relatively quiet background solar wind activity. During the entire interval of one month, we have identified 11 individual events of simultaneously increased magnetosheath particle populations, signatures of ion acceleration and magnetospheric compressions at Mars, out of which we have chosen to present three examples in this paper. However, we like to point out that all of these 11 
active periods, which lasted of the order of less than a day to at maximum two days, have been associated with short-lived intensifications of the solar wind dynamic pressure. Throughout the rest of the time, the particle data from the vicinity of Mars pretty much resembled a quiet time situation, with normal density and temperature of the magnetosheath electron and ion populations. To illustrate this, we have shown data from a very quiet solar wind period as an example for the characteristics of the quiet time Martian magnetosphere. Furthermore, for each of the presented active events, we show that (and how) the observed event-like disturbances develop out of, and subsequently recover to, a magnetospheric configuration resembling this quiet time state. We also note that these active periods occur for less than $30 \%$ of the time considering that we have seen 11 such intensifications on average less than a day long during a 30 day campaign period.

[80] As a side note, we would like to point out that, indeed, the original campaign was planned for 1-31 March 2010, based on conjunction predictions using normal solar wind velocities. After the campaign, we realized that the solar wind velocities had been lower than normal and thus the prime conjunction period instead had occurred from 15 March to 15 April, allowing us to include the CME event of 5-6 April. In retrospect, we also noticed that the period from 1 to 15 March 2010 contained only two very minor solar wind intensifications and corresponding disturbances in the Martian induced magnetosphere. Thus, our effective event occurrence rate should be around $20 \%$ instead, when looking at the entire campaign interval of 6 weeks.

[81] During these short-lived active intervals, we noted from the ASPERA-3 data particularly higher count rates and wider energy spread of electron and ion populations in the sheath, plus clear dayside and even flank bow shock compressions. Also and most prominent, we see that the acceleration of heavy ionospheric ions to suprathermal energies between several $10 \mathrm{~s}$ to several $100 \mathrm{~s}$ of eV only occurs during these short time intervals, possibly better explaining the statistically very close dependence on solar wind activity for the estimation of suprathermal heavy ion outflows, which have been presented by, e.g., Lundin et al. [2008b], and Nilsson et al. [2010]. In fact, it might even explain some of the discrepancies (by more than one order of magnitude) between estimated total ion outflow from Mars during various publications from the ASPERA team, as the solar activity during the period from which the statistics are collected appear to be very important. However, and as discussed further below, the lower range of ion energies observed by the ASPERA-3 instrument seems to play an even more important role in the estimation of total on outflow from the planet.

[82] Our observations also confirm in all cases that the acceleration of heavy ionospheric ions increases with increasing altitude, a detail which has also been described by Dubinin et al. [2006], and in two event studies for CIR-related solar wind disturbances by Edberg et al. [2009b] and Dubinin et al. [2009]. Both author teams attributed this - as we have found - very prominent feature in the observed heavy ion population under disturbed conditions to an acceleration by electric fields in the $-\mathbf{V} \times \mathbf{B}$ direction, imposed by the draped IMF in the Martian induced magnetosphere. Pérez-de-Tejada et al. [2009] have identified a similar case where the ionospheric ions above the polar region of
Mars close to the terminator were accelerated to energies well above that of the solar wind protons in the magnetosheath. They attributed such energization to viscous interaction of the ionosphere with the slowed down magnetosheath solar wind particles, forming a tailward flowing velocity boundary layer, in analogy to similar observations at Venus. As we have seen, and will discuss further below, all our events have been associated with an increase in the draped magnetic field as deduced by using the MARSIS AIS capability of detecting electron gyrolines in the local topside ionospheric plasma, indicating stronger electric field, but also stronger viscous interaction forces.

[83] Outside the discussed periods of solar wind activity the boundaries between light energetic solar wind/magnetosheath ions and heavy ionospheric ions are well defined and sharp, with basically no or very small features of suprathermal acceleration beyond the typical ionospheric energies. In this context, it is interesting to note again that during the extended quiet time period of about two days, we observed an ever increasing heavy ion population in the central nightside tail, with suprathermal energies above several tens of $\mathrm{eV}$, but remaining well below $100 \mathrm{eV}$. However, in this case, there were no indications of acceleration with increasing distance from the planet, as was the case for all of the more active events and periods. This may indicate a slow energization of ionospheric ions even during very quiet times, which is usually hard to identify under disturbed conditions when a more extreme scavenging of the upper atmosphere occurs due to increased pressure, stronger electric fields and higher solar wind velocities. Such energization independent of altitude, deep down into the nightside tail, could well be the result of viscous forcing as described by Pérez-de-Tejada et al. [2009]. The observations presented here may for the first time help to characterize the ground state of atmospheric loss from Mars without particular erosion driven by solar wind activity and variability.

[84] Looking at times directly following CIR-related pressure pulses, we can point out that the associated long lasting periods of increased solar wind velocities do not seem to affect the acceleration of heavy ions very much. The dynamic pressure (i.e., increase in both solar wind $n_{e}$ and $v$ ) seems to be the most controlling factor for ion acceleration, as the features of ion acceleration die out as soon as the CIR/ CME-related density increase has passed by Mars. Consequently, the events seem to be of longer duration for CMEs, where the increased solar wind plasma density remains a constant feature within the entire fast moving plasma bubble, i.e., the duration of the dynamic pressure pulse should be much longer for CME type of events. We also note that during the smaller CME encounter during orbit 7984 (in the second event section 3.3) we found the most pronounced dayside acceleration of dayside ionospheric ions. This might well be a particular feature of CME encounters, as it was so prominent in the dayside part of the orbit, and very different from the CIR-related cases. However, in this study, we have too few confirmed data concerning central CME ejecta to be very firm on this point.

[85] We note that most studies on ion outflow dependencies on solar wind variations have been statistical, with only a few exceptional event studies listed in the introduction section. We believe that our study of multiple isolated events can in more detail identify the main processes and drivers for the acceleration of ionospheric ions, and thus help to better 
understand the overall loss of material from the upper atmosphere of the planet.

[86] As discussed in the introduction section, the fact that various statistical studies [Barabash et al., 2007; Lundin et al., 2008a; Nilsson et al., 2010] of total ion outflow from Mars - using data from the same instrument - come to vastly different results concerning total ion outflow rates has partially been attributed to the various disturbance levels in the solar wind, and thus variable erosion of the Martian atmosphere during the years for which the statistics had been derived. However, as those authors particularly point out, the exact inclusion of the lowest suprathermal energy range of ionospheric heavy ions may even play a more important role in the exact determination of the average total ion outflow. So not surprisingly the inclusion of a lower energy limit of the detection range of the ASPERA-3 instrument by Lundin et al. [2008a], the additional careful interpretation of directional characteristics of the instrument by Nilsson et al. [2010], and recently even the inclusion of information about the thermal ionospheric plasma from MARSIS AIS by Fränz et al. [2010] have led to higher and higher values of the estimated total ion outflow, now ranging somewhere between $10^{24} \mathrm{~s}^{-1}$ and $10^{25} \mathrm{~s}^{-1}$.

[87] In this study, we systematically include combined ASPERA-3 observations of the energetic plasma and MARSIS AIS observations of the cold ionospheric plasma in an attempt to understand the response of the Martian ionosphere/magnetosphere system to solar wind variability. During this campaign, we have achieved an unprecedented cadence of consecutive and full range low orbit operations of the MARSIS AIS instrument, and as such our data are already somewhat unique in the history of the Mars Express mission. Earlier studies only discussed individual orbits with very scarce or no MARSIS AIS data coverage [Dubinin et al., 2008a,2008b; Duru et al., 2009; Fränz et al., 2010; Dubinin et al., 2011].

[88] In more detail, we have established a few new observations in association with Solar wind variations on the Martian ionosphere. Using the capability of MARSIS AIS to determine local plasma parameters such as electron density and total magnetic field, we could show that during all events of solar wind pressure increase the dayside ionosphere disappears below the altitude of the spacecraft orbit. We can see how the intrusion of fast moving low-density magnetosheath plasma suddenly inhibits the detection of local plasma lines at the MARSIS Antenna and thus the AIS instrument's capability to estimate the local electron density. This sudden disappearance of the local upper ionosphere has earlier been reported for one CIR encounter by Dubinin et al. [2009] and was interpreted as an extreme scavenging of the topside ionosphere by increased solar wind erosion. In contrast, we would like to interpret such MARSIS AIS observations as a compression of the ionosphere to altitudes below the spacecraft orbit, as it is in very good agreement with the simultaneous ASPERA observations of compressions of the dayside boundary between high-energy sheath and low-energy ionospheric electrons and ions, and also often the sudden appearance of energized heavy ions below that compressed boundary. Furthermore, from the AIS data on the nightside of the terminator, we see that the electron density most of the time increases there during such events, which could be explained by a flaring of the nightside ionosphere in response to a similar dayside compression. In addition, we note that the capability of AIS to detect electron gyrolines does not vanish as fast as the detection of plasma-line echoes during these events, and thus we could deduce that during these compression events the total draped magnetic field strength on the dayside of Mars generally increased by a factor of about 1.5 to 2. This appears to be a large enough pressure variation to move the MPB to below the MEX spacecraft. We note in this context that also Brain et al. [2005], using MGS electron spectrometer data, found ionospheric compressions, such that magnetosheath plasma was observed by MGS at $400 \mathrm{~km}$ altitude, for up to $20 \%$ of the time, which is in good agreement with our "event occurrence rate" during this campaign.

[89] As a final point, we would like to discuss that our AIS observations can also demonstrate an important effect on previous attempts to include MARSIS data concerning thermal topside plasma densities, together with simultaneous ASPERA data, in the calculation of total loss rates from the Martian atmosphere, as recently presented by Fränz et al. [2010]. While there is no doubt from the work of Fränz and coworkers that thermal plasma losses are very important for the overall calculations of upper atmosphere erosion by the solar wind, one must use care in the interpretation of MARSIS AIS data during disturbed times, when the solar wind dynamic pressure is particularly high.

[90] As we have shown, the use of MARSIS topside electron densities to complement ASPERA-3 low-energy ion data will obviously work very well outside periods of solar wind pressure increases, and thus then and there the extra thermal element of ion outflow during quiet times can be important. However, our observations show that during disturbed times the dayside ionosphere can be compressed to below the spacecraft ionosphere at the same time as heavy ion acceleration as seen by ASPERA-3 increases. Thus, during solar wind activity ASPERA-3 will see an increase in the energetic particle outflow. But to automatically include thermal electron densities from MARSIS AIS in situ data in ionospheric outflow calculations could lead to a severe underestimation of the thermal outflow, when the ionosphere is suddenly depressed below spacecraft altitude. In some preliminary and very crude outflow integration attempts, using data from our individual events, we have seen an apparent decrease of total outflow rates during solar wind pressure pulses, caused by this effect. As such behavior would be quite unphysical, great care needs to be taken in how and from where one uses MARSIS AIS in situ data to determine thermal ionospheric densities, when the topside ionosphere appears to be heavily deformed by solar wind activity. This could even effect statistical studies, if many compression events fall into the period, the energetic outflow might be seen as dominant and the thermal outflow underestimated, potentially leading to misleading results for total ion outflows during active times.

\section{Conclusions}

[91] We have shown that, indeed, solar wind data from $1 \mathrm{AU}$ can be used to determine solar wind variability at Mars, at least for solar wind conditions involving CIRs leading to solar wind structures with Parker spiral geometry. For CME-related activity, additional STEREO HI or other remote sensing observation techniques are required to address the exact shape and travel path of the solar ejecta. During this 
6 weeks long campaign period during close to solar minimum conditions, we have found a one-to-one relation between solar wind pressure pulses (caused by various solar wind phenomena like CIR, CME, etc.) to magnetospheric compressions and increased acceleration of ionospheric ions at Mars, seen by ASPERA-3. During this campaign, data were taken with the MARSIS instrument at a higher cadence than typical, since this proved to be very useful in characterizing the behavior of the thermal topside ionosphere and the amount of magnetic draping during these compression events. However, consecutive full low orbit passage MARSIS AIS operations for more than five orbits in sequence would have been needed to exploit the power of the combined ASPERA-3 and MARSIS data to its full extent.

[92] We also found that the combination of simultaneously increased velocity and increased solar wind density (i.e., dynamic pressure) was a more efficient driver for magnetospheric/ionospheric responses at Mars than velocity or density by itself. Except for the longer duration of CME-related events, which is probably related to the longer duration of the CME ejecta passing by the planet, there was no characteristic difference between CIR and CME-related pressure variations in the response of Mars. But one clear CME (which hit Mars only with its western flank) and one suspected CME arriving at the time of an already ongoing CIR event do not allow us to draw deeper conclusions on this question. At the time of writing this paper, we already have first access to data from a new campaign, which in the spring of 2012 during the fast rising new solar cycle has resulted in the observation of far more CME-related events at Mars. This data will be analysed by an ISSI team in the near future, and published thereafter in due course.

[93] Acknowledgments. The dedicated effort leading to this paper would not have been possible without input from the entire MUAN network of scientists and help from the ESA Mars-Express project team, the entire Science Operation Working Group, and the benevolent understanding and collegial behavior of the nonplasma PIs and scientists of the MEX mission, in particular the MARSIS subsurface sounder team, which has given up observation time for the benefit of this dedicated campaign. We also wish to thank Ruth Skoug for helpful discussions regarding the Wind data used in this study.

[94] The work of HO, DA, and NJTE has been made possible by grants from the Swedish National Space Board, SNSB. HO also acknowledges the peaceful and fruitful environment of ISSI for the detailed analysis of data and finalization of this manuscript during repeated visits in 2012 and 2013. M.F. would like to thank the German Space Agency for support through grant DLR50QM080 and the German Science Foundation through grant DFG WO910/3-1. ML acknowledges support from STFC grant ST/H002840/1.

[95] Masaki Fujimoto thanks Héctor Pérez de Tejada Jaime and Robert Lillis for their assistance in evaluating this paper.

\section{References}

Allen, J. (2010), The Galaxy 15 Anomaly: Another Satellite in the Wrong Place at a Critical Time, Space Weather, 8, S06008, doi:10.1029/ 2010SW000588.

Barabash, S., et al. (2006), The Analyzer of Space Plasmas and Energetic Atoms (ASPERA-3) for the Mars Express Mission, Space Sci. Rev., 126, 113-164, doi:10.1007/s11214-006-9124-8.

Barabash, S., A. Fedorov, R. Lundin, and J. J. Sauvaud (2007), Martian Armospheric erosion rates, Science, 315, 501

Bertucci, C., et al. (2003) Magnetic field draping enhancement at the Martian magnetic pileup boundary from Mars global surveyor observations, Geophys. Res. Lett., 30(2), 1099, doi:10.1029/2002GL015713.

Brain, D. A., J. S. Halekas, R. Lillis, D. L. Mitchell, R. P. Lin, and D. H. Crider (2005) Variability of the altitude of the Martian sheath, Geophys. Res. Lett., 32, L18203, doi:10.1029/2005GL023126,

Cain, J. C., B. B. Ferguson, and D. Mozzoni (2003), An $n=90$ internal potential function of the Martian crustal magnetic field, J. Geophys. Res., 108(E2), 5008, doi:10.1029/2000JE001487.
Chicarro, A., P. Martin, and R. Trautner (2004), The Mars Express mission: An overview, in Mars Express: The Scientific Payload, ESA Special Publication, vol. 1240, edited by A. Wilson and A. Chicarro, pp. 3-13, European Space Agency, Noordwijk, The Netherlands.

Dubinin, E., et al. (2006), Electric fields within the Martian magnetosphere and ion extraction: ASPERA-3 observations, Icarus, 182, 337-342, doi:10.1016/j.icarus.2005.05.022.

Dubinin, E., et al. (2008a), Plasma environment of Mars as observed by simultaneous MEX-ASPERA-3 and MEX-MARSIS observations, J. Geophys. Res., 113, A10217, doi:10.1029/2008JA013355.

Dubinin, E., et al. (2008b), Structure and dynamics of the solar wind/ ionosphere interface on Mars: MEX-ASPERA-3 and MEX-MARSIS observations, Geophys. Res. Lett., 35, L11103, doi:10.1029/ 2008GL033730.

Dubinin, E., M. Fränz, J. Woch, F. Duru, D. Gurnett, R. Modolo, S. Barabash, and R. Lundin (2009), Ionospheric storms on Mars: Impact of the corotating interaction region, Geophys. Res. Lett., 360, L01105, doi:10.1029/2008GL036559.

Dubinin, E., M. Fränz, A. Fedorov, R. Lundin, N. Edberg, F. Duru, and O. Vaisberg (2011), Ion Energization and Escape on Mars and Venus, Space Sci. Rev., 162, 173-211, doi:10.1007/s11214-011-9831-7.

Duru, F., D. A. Gurnett, D. D. Morgan, R. Modolo, A. F. Nagy, and D. Najib (2008), Electron densities in the upper ionosphere of Mars from the excitation of electron plasma oscillations, J. Geophys. Res., 113, A07302, doi:10.1029/2008JA013073.

Duru, F., D. A. Gurnett, R. A. Frahm, J. D. Winningham, D. D. Morgan, and G. G. Howes (2009), Steep, transient density gradients in the Martian ionosphere similar to the ionopause at Venus, J. Geophys. Res., 114, A12310, doi:10.1029/2009JA014711.

Edberg, N. J. T., D. A. Brain, M. Lester, S. W. H. Cowley, R. Modolo, M. Fränz, and S. Barabash (2009a), Plasma boundary variability at Mars as observed by Mars Global Surveyor and Mars Express, Ann. Geophys., 27, 3537-3550, doi:10.5194/angeo-27-3537-2009.

Edberg, N. J. T., et al. (2009b), Rosetta and Mars Express observations of the influence of high solar wind pressure on the Martian plasma environment, Ann. Geophys., 27, 4533-4545, doi:10.5194/angeo-27-4533-2009.

Edberg, N. J. T., H. Nilsson, A. O. Williams, M. Lester, S. E. Milan, S. W. H. Cowley, M. Fränz, S. Barabash, and Y. Futaana (2010), Pumping out the atmosphere of Mars through solar wind pressure pulses, Geophys. Res. Lett., 370, L03107, doi:10.1029/2009GL041814.

Eyles, C. J., et al. (2009), The Heliospheric Imagers Onboard the STEREO Mission, Sol. Phys., 254, 387-445, doi:10.1007/s11207008-9299-0.

Fränz, M., et al. (2006), Plasma intrusion above Mars crustal fields: Mars Express ASPERA-3 observations, Icarus, 182, 406-412, doi:10.1016/j. icarus.2005.11.016.

Fränz, M., E. Dubinin, E. Nielsen, J. Woch, S. Barabash, and R. Lundin (2010), Transterminator ion flow in the Martian ionosphere, Planet. Space Sci., 58(11), 1442-1454, doi:10.1016/j.pss.2010.06.009.

Futaana, Y., et al. (2008), Mars Express and Venus Express multi-point observations of geoeffecive solar flare events in Dcember 2006, Planet. Space Sci., 56, 873-880, doi:10.1016/j.pss.2007.10.014.

Gurnett, D. A., et al. (2005), Radar Soundings of the Ionosphere of Mars, Science, 310, 1929-1933, doi:10.1126/science.1121868.

Jordan, R., et al. (2009), The Mars Express MARSIS sounder instrument, Planet. Space Sci., 57, 1975-1986, doi:10.1016/j.pss.2009.09.016.

Lammer, H., et al. (2013), Outgassing History and Escape of the Martian Atmosphere and Water Inventory, Space Sci. Rev., 174, 113-154, doi:10.1007/s11214-012-9943-8.

Lepping, R. P., et al. (1995), The Wind Magnetic Field Investigation, Space Sci. Rev., 71, 207-229, doi:10.1007/BF00751330.

Lillis, R. J., M. O. Fillingim, and D. A. Brain (2011), Three-dimensional structure of the Martian nightside ionosphere: Predicted rates of impact ionization from Mars Global Surveyor magnetometer and electron reflectometer measurements of precipitating electrons, J. Geophys. Res., 116, A12317, doi:10.1029/2011JA016982.

Lundin, R., S. Barabash, M. Holmström, H. Nilsson, M. Yamauchi, M. Fränz, and E. M. Dubinin (2008a), A comet-like escape of ionospheric plasma from Mars, Geophys. Res. Lett., 351, L18203, doi:10.1029/ 2008 GL034811.

Lundin, R., S. Barabash, A. Fedorov, M. Holmström, H. Nilsson, J.-A. Sauvaud, and M. Yamauchi (2008b), Solar forcing and planetary ion escape from Mars, Geophys. Res. Lett., 35, L09203, doi:10.1029/2007GL032884.

Morgan, D. D., D. A. Gurnett, D. L. Kirchner, J. L. Fox, E. Nielsen, and J. J. Plaut (2008), Variation of the Martian ionospheric electron density from Mars Express radar soundings, J. Geophys. Res., 113, A09303, doi:10.1029/2008JA013313.

Morgan, D. D., et al. (2010), Radar absorption due to a corotating interaction region encounter with Mars detected by MARSIS, Icarus, 206, 95-103, doi:10.1016/j.icarus.2009.03.008. 


\section{OPGENOORTH ET AL.: MARS IONOSPHERIC RESPONSE}

Nilsson, H., E. Carlsson, D. A. Brain, M. Yamauchi, M. Holmström, S. Barabash, R. Lundin, and Y. Futaana (2010), Ion escape from Mars as a function of solar wind conditions: A statistical study, Icarus, 206 40-49, doi:10.1016/j.icarus.2009.03.006.

Odstrcil, D., and V. J. Pizzo (1999), Distortion of the interplanetary magnetic field by three-dimensional propagation of coronal mass ejections in a structured solar wind, J. Geophys. Res., 1042, 28,225-28,240, doi:10.1029/1999JA900319.

Odstrcil, D., and V. J. Pizzo (2009), Numerical Heliospheric Simulations as Assisting Tool for Interpretation of Observations by STEREO Heliospheric Imagers, Sol. Phys., 259, 297-309, doi:10.1007/s11207009-9449-z.

Ogilvie, K. W., et al. (1995), SWE, A Comprehensive Plasma Instrument for the Wind Spacecraft, Space Sci. Rev., 71, 55-77, doi:10.1007/ BF00751326.

Pérez-de-Tejada, H., R. Lundin, H. Durnad-Manterola, and M. Reyes-Ruiz (2009), Solar wind erosion of the polar regions of the Mars ionosphere, J. Geophys. Res., 114, A02106, doi:10.1029/2008JA013295.

Rouillard, A. P., et al. (2010), Intermittent release of transients in the slow solar wind: 1. Remote sensing observations, J. Geophys. Res., 115, A04103, doi:10.1029/2009JA014471.
Sheeley, N. R., J. H. Walters, Y.-M. Wang, and R. A. Howard (1999), Continuous tracking of coronal outflows: Two kinds of coronal mass ejections, J. Geophys. Res., 104, 24,739-24,768, doi:10.1029/1999JA900308. Tsurutani, B. T., W. D. Gonzalez, A. L. C. Gonzalez, F. Tang, J. K. Arballo, and M. Okada (1995), Interplanetary origin of geomagnetic activity in the declining phase of the solar cycle, J. Geophys. Res., 100, 21,717-21,733. Vennerstrøm, S., N. Olsen, M. Purucker, M. H. Acuña, and J. C. Cain (2003), The magnetic field in the pile-up region at Mars, and its variation with the solar wind, Geophys. Res. Lett., 30(7), 1369, doi:10.1029/ 2003GL016883.

Vignes, D., et al. (2000), The solar wind interaction with Mars: Locations and shapes of the Bow Shock and the magnetic pile-up boundary from the observations of the MAG/ER experiment onboard Mars Globa Surveyor, Geophys. Res. Lett., 27, 49-52, doi:10.1029/1999GL010703.

Wei, Y., et al. (2012), Enhanced atmospheric oxygen outflow on Earth and Mars driven by a corotating interaction region, J. Geophys. Res., 117, A03208, doi:10.1029/2011JA017340.

Williams, A. O., N. J. T. Edberg, S. E. Milan, M. Lester, M. Fränz, and J. A. Davies (2011), Tracking corotating interaction regions from the Sun through to the orbit of Mars using ACE, MEX, VEX, and STEREO, J. Geophys. Res., 116, A08103, doi:10.1029/2010JA015719. 